University of Louisville

ThinkIR: The University of Louisville's Institutional Repository

Electronic Theses and Dissertations

$11-2019$

\title{
A kinetic and thermodynamic model of ethylene dichloride pyrolysis.
}

Travis J Czechorski

University of Louisville

Follow this and additional works at: https://ir.library.louisville.edu/etd

Part of the Catalysis and Reaction Engineering Commons, Thermodynamics Commons, and the Transport Phenomena Commons

\section{Recommended Citation}

Czechorski, Travis J, "A kinetic and thermodynamic model of ethylene dichloride pyrolysis." (2019).

Electronic Theses and Dissertations. Paper 3359.

https://doi.org/10.18297/etd/3359

This Master's Thesis is brought to you for free and open access by ThinkIR: The University of Louisville's Institutional Repository. It has been accepted for inclusion in Electronic Theses and Dissertations by an authorized administrator of ThinkIR: The University of Louisville's Institutional Repository. This title appears here courtesy of the author, who has retained all other copyrights. For more information, please contact thinkir@louisville.edu. 


\author{
Travis James Czechorski \\ B.S., University of Louisville, 2018 \\ A Thesis \\ Submitted to the Faculty of the \\ Speed School of Engineering of the University of Louisville \\ in Partial Fulfillment of the Requirements \\ for the Degree of \\ Master of Engineering (M.Eng.) \\ in Chemical Engineering \\ Department of Chemical Engineering \\ University of Louisville \\ Louisville, Kentucky
}

November 2019 
A KINETIC AND THERMODYNAMIC MODEL OF ETHYLENE DICHLORIDE PYROLYSIS

By

Travis James Czechorski

B.S., University of Louisville, 2018

A Thesis Approved on

November 25, 2019

by the following Thesis Committee:

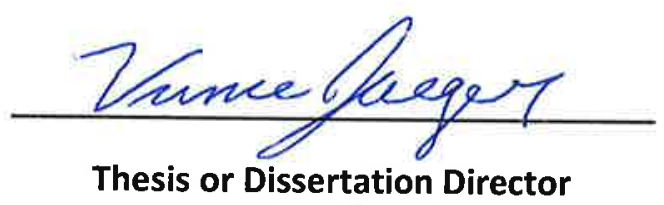

Vance $W$ Jaeger

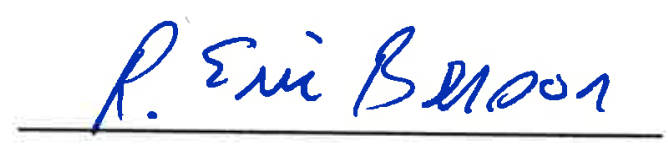

Eric Benson


Badri Narayanan

iii 


\begin{abstract}
A KINETIC AND THERMODYNAMIC MODEL OF ETHYLENE DICHLORIDE PYROLYSIS Travis Czechorski

November 19th, 2019

Ethylene dichloride (EDC) is a precursor for the production of vinyl chloride (VCM) which is subsequently polymerized to form polyvinyl chloride (PVC). To convert EDC to VCM, EDC undergoes a pyrolysis process in the absence of oxygen at temperatures exceeding 500C. However, process yields are limited by the uncontrolled production of side-products that can degrade the quality of PVC and poison the reactor. Thus, tight process controls and costly separations guided by heuristics and plant operator experience are used to optimize EDC pyrolysis. To improve the process, I have programed a kinetic model of EDC pyrolysis based upon estimations of kinetic and thermodynamic parameters for known products and sideproducts. These parameters are activation energy over a range of $342.0-317.0 \mathrm{~kJ} / \mathrm{mol}$ and temperature over a range of 450-650 C. With this model, I have predicted the effects of process temperature and feed composition. However, due to high process temperatures and the intense environment in which EDC is converted to VCM, model parameters cannot be measured experimentally in most cases and must instead be estimated by various theoretical means. To address possible weaknesses in the methods used to estimate model parameters, I have calculated the effects of their misestimation and characterized model sensitivity. I aim to guide future research toward improved methods for kinetic and thermodynamic property estimation. I plan to generalize our method to apply to any complex reaction network so that the scientific and engineering community can discover targets for catalysis research.
\end{abstract}




\section{Table of Contents}

List of Figures




\section{List of Figures}

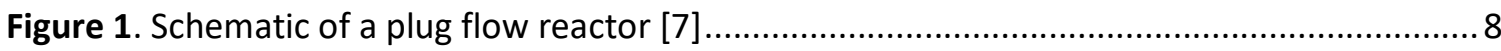

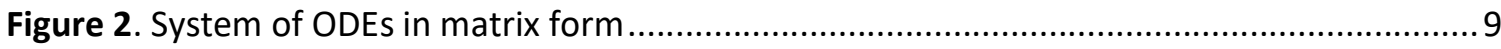

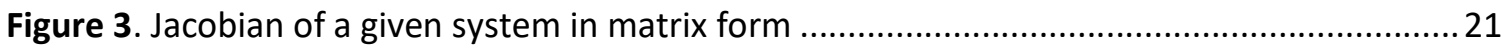

Figure 4. Code used to symbolically calculate the Jacobian ........................................................22

Figure 5. Concentration profile of EDC, $\mathrm{HCl}$, and $\mathrm{VCM}$ at the initial operating conditions .......... 25

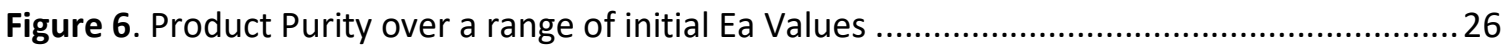

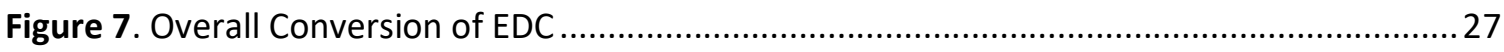

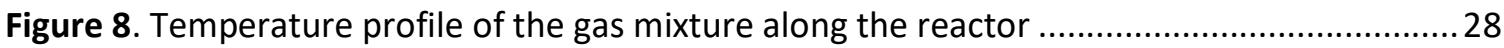



Figure 10. Concentration profile of EDC, $\mathrm{HCl}$, and VCM with an initial Ea of $337.0 \ldots \ldots \ldots \ldots \ldots \ldots . . . . . . . . . . .30$

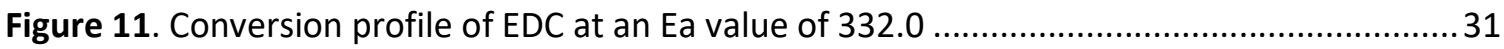

Figure 12. Concentration profile of $\mathrm{EDC}, \mathrm{HCl}$, and VCM with an initial $\mathrm{Ea}$ of $332.0 \ldots \ldots \ldots \ldots \ldots . . . . . . . . .31$

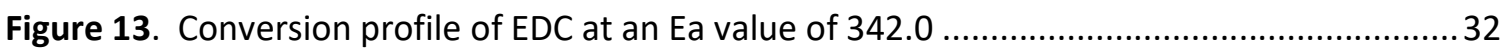

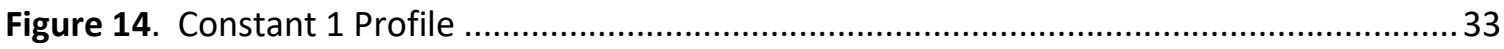

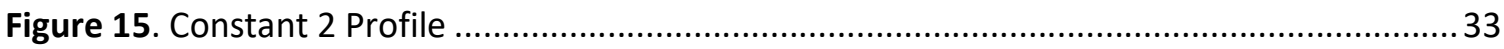

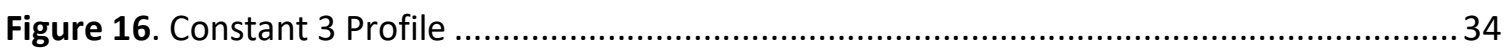

Figure 17. Product Concentration profile with an initial temperature of $923.15 \mathrm{~K} \ldots \ldots \ldots \ldots \ldots \ldots . . . . . . . . . .35$

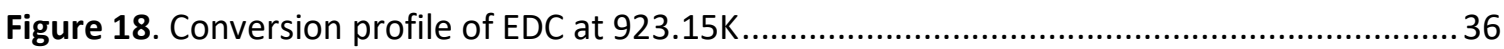



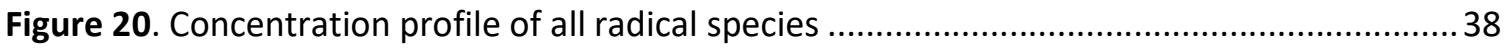

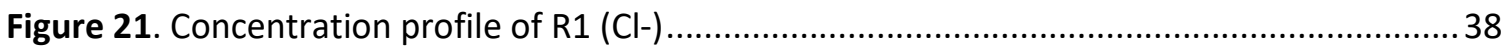

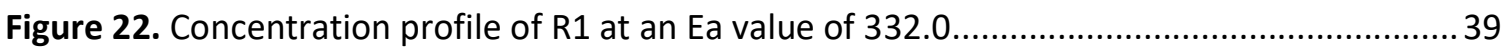



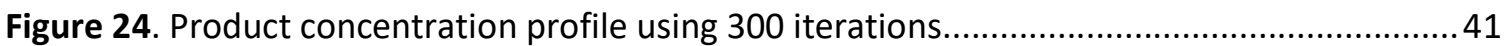

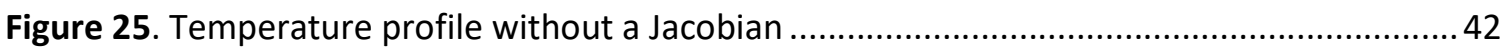



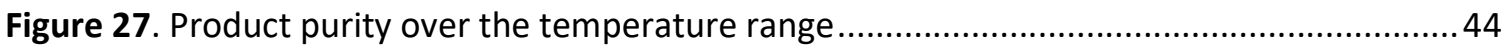

Figure 28. Conversion of EDC over the activation energy range .............................................. 44 


\section{Introduction}

Polyvinyl chloride (PVC) is a ubiquitous plastic material used in construction, infrastructure and housing among many other applications. PVC is produced by the polymerization of vinyl chloride monomer (VCM), and there are three main methods to manufacture VCM. The three methods are pyrolysis of ethylene dichloride (EDC), direct chlorination of ethylene, and oxychlorination of ethylene. Of the three methods, pyrolysis of EDC is the most widely utilized in the industrial sector. This reaction is endothermic and most often takes place in a tubular plug flow reactor (PFR). However, if certain process variables are not kept under tight control, significant amounts of unwanted side products start to form. These side products are both expensive to remove from the process stream and dangerous to the environment and workers. Moreover, they can shorten equipment lifespan and increase maintenance costs. Due to these facts, the conversion of EDC to VCM is usually kept around $60 \%$ for a single pass in a reactor to minimize side product formation. This process constraint forces PVC producers to purify large volumes of process chemical to return unreacted EDC to the reactor, and controls must be rigorously implemented to maintain an efficient, profitable process. There is therefore great interest in discovering methods and procedures to increase EDC conversion without increasing side product formation. 
Typically, EDC cracking is performed at temperatures ranging from 773.15 923.15 K [1]. At these temperatures, there are a multitude of reactions taking place, with only a few leading to useful products. The kinetics of each reaction are difficult to measure experimentally due to high temperatures, short-lived intermediates, and coking of experimental equipment. Previously published studies have compiled sets of preexponential Arrhenius constants and activation energies for the EDC cracking process based on experimental measurements and theoretical calculations. Because of the large reaction network and differences in several orders of magnitude in reaction rates, the EDC cracking process is difficult to model. When designing a model for this reaction process, there are two main parts taken into consideration, namely the mass balance and the energy balance. These two balances guide this study and can be used to predict the effects of changes to process variables, including flow rates, temperatures, and the addition of hypothetical catalysts or inhibitors. The model will be used to hypothesize methods or modifications to existing process to increase the conversion EDC to VCM without increasing the production of unwanted side products.

EDC cracking is a multistep reaction that begins by the conversion of EDC to two radical species $\mathrm{R}_{1}\left(\mathrm{Cl}^{-}\right)$and $\mathrm{R}_{2}\left(\mathrm{CH}_{2} \mathrm{ClCH}_{2}{ }^{-}\right)$. There is great interest in designing a catalyst to reduce the activation energy of this initiating reaction without increasing the rate of production for the by-products. However, efforts to produce a stable, long-lived catalyst for EDC cracking remain unfruitful. The value of a hypothetical catalyst depends heavily upon how far it can lower the activation energy of the initiating reaction, and the reaction will be modeled both without catalyst and with catalysts of varying strength. 
Reaction kinetics are modeled with the Arrhenius equation which requires an activation energy and a pre-exponential frequency factor at a specific reactor temperature. Reaction order and stoichiometry also play a significant role in system behavior. Each of these variables can cause results to vary by orders of magnitude, especially with respect to the production of byproduct species. Moreover, as the modeled reaction network grows in terms of reactions and number of species, models become increasingly difficult to program and utilize. Eventually, an analytical solution to the reactor design equations becomes untenable, and numerical solutions remain the only viable method of solving these problems. Some of the main challenges faced when attempting to accurately model the EDC cracking process involve numerical methods and the amount of computer memory and speed needed to store and process numbers that have many significant digits, and values spanning several orders of magnitude. Even when using numerical techniques to find an approximate solution, numerical errors and convergence may become significant issues. In situations like this choice of numerical solving method can make a significant difference. If a system of ODEs slowly converges or simply does converge, the system is said to be "stiff". A stiff system of ODEs is generally resistant to many methods of numerical integration, and special methods must be employed to receive an answer [2].

In total over 800 individual reactions and over 135 individual chemical species have been identified and studied in the EDC pyrolysis reaction network [3]. In order to produce a working model, simplifications have to be made to reduce the number of species modeled. Previous models developed by Schirmeister and coworkers [1] used 31 


\begin{tabular}{|c|c|c|c|}
\hline \multicolumn{4}{|c|}{ Table 1. Reactions for the Simple model } \\
\hline Number & Reaction & $E_{a}(\mathrm{~kJ} / \mathrm{mol})$ & $k_{0}\left[\left(\mathrm{~cm}^{3} / \mathrm{mol}\right)^{n-1}\right] / s$ \\
\hline 1 & $\mathrm{EDC} \rightarrow R_{1}+R_{2}$ & 342.0 & $5.900 \mathrm{e}+15$ \\
\hline 2 & $\mathrm{EDC}+R_{1} \rightarrow \mathrm{HCl}+R_{3}$ & 7.0 & $1.300 \mathrm{e}+13$ \\
\hline 3 & $\mathrm{EDC}+R_{2} \rightarrow \mathrm{EC}+R_{3}$ & 42.0 & $1.000 \mathrm{e}+12$ \\
\hline 4 & $\mathrm{EDC}+R_{4} \rightarrow 1,1$-dichloroethane $+R_{3}$ & 45.0 & $5.000 \mathrm{e}+11$ \\
\hline 5 & $\mathrm{EDC}+R_{5} \rightarrow \mathrm{VCM}+R_{3}$ & 34.0 & $1.200 \mathrm{e}+13$ \\
\hline 6 & $\mathrm{EDC}+R_{6} \rightarrow 1,1,2$-trichloroethane $+R_{3}$ & 48.0 & $2.000 \mathrm{e}+11$ \\
\hline 7 & $R_{1}+R_{2} \rightarrow \mathrm{VCM}+\mathrm{HCl}$ & 13.0 & $1.000 \mathrm{e}+13$ \\
\hline 8 & $R_{1}+R_{3} \rightarrow 1,1-/$ cis-/trans-dichloroethylene $+\mathrm{HCl}$ & 12.0 & $1.000 \mathrm{e}+13$ \\
\hline 9 & $R_{1}+\mathrm{EC} \rightarrow \mathrm{HCl}+R_{2}$ & 4.0 & $1.700 \mathrm{e}+13$ \\
\hline 10 & $R_{1}+1,1-$ dichloroethane $\rightarrow \mathrm{HCl}+R_{4}$ & 6.0 & $1.200 \mathrm{e}+13$ \\
\hline 11 & $R_{1}+1,1,2$-trichloroethane $\rightarrow \mathrm{HCl}+R_{6}$ & 15.0 & $1.700 \mathrm{e}+13$ \\
\hline 12 & $\mathrm{VCM}+R_{1} \rightarrow R_{4}$ & 0.0 & $9.100 \mathrm{e}+10$ \\
\hline 13 & $\mathrm{VCM}+R_{1} \rightarrow \mathrm{HCl}+R_{5}$ & 56.0 & $1.200 \mathrm{e}+14$ \\
\hline 14 & $\mathrm{VCM}+R_{2} \rightarrow \mathrm{EC}+R_{5}$ & 31.0 & $5.000 \mathrm{e}+11$ \\
\hline 15 & $\mathrm{VCM}+R_{4} \rightarrow C_{4} H_{6} C l_{2}+R_{1}$ & 30.0 & $2.000 \mathrm{e}+10$ \\
\hline 16 & $\mathrm{VCM}+R_{5} \rightarrow \mathrm{CP}+R_{1}$ & 61.0 & $3.000 \mathrm{e}+11$ \\
\hline 17 & $R_{3} \leftarrow \rightarrow \mathrm{VCM}+R_{1}$ & 84.0 & $2.100 \mathrm{e}+14$ \\
\hline 18 & $R_{5} \leftarrow \rightarrow C_{2} H_{2}+R_{1}$ & 90.0 & $5.000 \mathrm{e}+14$ \\
\hline 19 & $R_{6} \leftarrow \rightarrow 1,1-/$ cis-/trans-dichloroethylene $+R_{1}$ & 70.0 & $2.000 \mathrm{e}+13$ \\
\hline 20 & $2 * C_{2} H_{2}+R_{5} \rightarrow C_{6} H_{6}+R_{1}$ & 20.0 & $1.000 \mathrm{e}+14$ \\
\hline 21 & $C_{2} H_{2}+2 * R_{1} \rightarrow 2 * C+2 * H C l$ & 70.0 & $1.600 \mathrm{e}+14$ \\
\hline
\end{tabular}

Table 1. All values are obtained from reference [1]

elementary reactions in total. Carbon tetrachloride $\left(\mathrm{CCl}_{4}\right)$ is included among the Schirmeister's reactants, because it can more readily produce the radicals needed to initiate the chain reaction, and it can significant effects on the system at very small initial concentrations. Carbon tetrachloride increases conversion significantly but also increases the production of unwanted side products. Carbon tetrachloride is often completely absent from the process, which further simplifies Schirmeister's reaction network. Two reaction networks were built for the models presented herein. The first, the simple model, has 21 elementary reactions. The second, full model will has all of Schirmeister's 31 elementary reaction equations. [1] These elementary reactions will coupled to a mass and energy balances to track the concentration of all species over the reactor's length. Activation energies and frequency factors are taken from [1]. As is 
shown in the table below, the activation energy for the initial reaction is nearly an order of magnitude higher than all other reactions. One of the main uses of the developed models will be to simulate the effects of changing the activation energy of the initial reaction to computationally determine the effects a catalyst might have on process yields. Initial pressures are typically somewhere in the range of 12-20 bar [1] [4] with initial temperatures around 773.15 K. [1] An initial value of 12 bar was used for the entirety of this experiment.

Once the initial reaction is initiated, it immediately forms 2 radicals $\left(R_{1} \& R_{2}\right)$. All subsequent reactions have a significantly higher reaction rate, and therefore the system quickly becomes complex. It is thought that the radical $\mathrm{R}_{1}$ could be artificially introduced to the system, process temperatures could be significantly reduced, thus reducing side product formation. $R_{1}$ is a necessary radical on the reaction path from EDC to VCM and $\mathrm{HCl}$, however as radicals are very short lived and exist in small quantities, it is hard to accurately predict the effect of $\mathrm{R}_{1}$ without kinetic modelling.

The most important process control parameter is temperature. In some cases, the conversion is directly proportional to temperature. [5] However, due to the complexities of heat and mass transfer equations in two and three dimensions, they are often difficult to accurately solve. Analytical solutions to the reactor design equations coupled with thermodynamics and kinetics in higher dimensionalities either rarely exist or depend on specific boundary conditions or assumptions that represent the real system. For the purpose of this paper, all differential questions will be steady-state and in 1-dimension (i.e. the length of the tubular reactor). While partial differential equations (PDE) solvers 
do exist, in most cases, they tended to be too complex or to not fit the specific requirements demanded by the solvers. Mass and heat equations were coupled together in the ODE system and solved numerically to simulate an EDC cracking process.

The model for this simulation was built using the Python programing language. Python is an open-source high-level programming language and is used extensively in data science and data engineering. Due to its extensive use, it has many open source libraries available for a wide variety of applications. Python is available on all major operating systems and it contains cross-language compilers for when extra speed or precision is required. Other languages such as $\mathrm{C}, \mathrm{C}++$, and FORTRAN have significantly higher computational speed, but lack many of the packages required to build and solve this reactor model. A goal of this project is to release and update the code to be freely used by any academic group, and therefore adopting a widely used and open source programming language makes sense to increase the impact of this work. Python is therefore an ideal choice for the EDC cracking reactor problem. Overall, I plan to accomplish the following objectives:

1. Design system of ODEs that accurately represent the system

2. Properly calculate all necessary coefficients and properly derive all energy and mass balance equations

3. Numerically integrate the system over the specified range for both activation energy and temperature 


\section{Methods}

The following equations represent the zeroth, first, second, and third order reaction rate laws. [6] The constant specific reaction rate constant ( $\mathrm{k}$ ) has varying units depending on the order of the rate law of a chemical species (i). [6]

Zeroth Order rate law:

$-\boldsymbol{r}_{i}=k \quad k=\mathrm{mol} /(\mathrm{dm} 3 * s)$

First Order rate law:

$-\boldsymbol{r}_{i}=k C_{i}$

$k=1 / s$

Second Order rate law:

$-\boldsymbol{r}_{\boldsymbol{i}}=k C_{i}^{2}$

$k=d m 3 /(\operatorname{mol} * s)$

Third Order rate law:

$-\boldsymbol{r}_{\boldsymbol{i}}=k C_{i}^{3}$

$k=[(d m 3 / m o l) 2] / s$

Rate order is determined by the number of reactants and their respective stoichiometry. If the exponents of the reactants in the rate law are identical to the stoichiometric coefficients of the reactants, the reaction is said to be elementary. [6] In terms of the pyrolysis of EDC, all considered reactions are assumed to be elementary. The reverse rate law is simply the opposite of the forward rate law, it is the concentration(s) of the products. For the case of an arbitrary chemical reaction as follows: 


$$
\mathrm{A} \leftrightarrows \mathrm{B}+\mathrm{C}
$$

The forward rate law for A would be:

$$
-\boldsymbol{r}_{A, \text { forward }}=k_{\text {forward }} C_{A}
$$

The reverse rate law for $A$ would be:

$$
\boldsymbol{r}_{\text {A,reverse }}=k_{\text {rervese }} C_{B} C_{C}
$$

This would give the overall rate law for a as:

$$
\boldsymbol{r}_{A}=\boldsymbol{r}_{A, \text { forward }}+\boldsymbol{r}_{A, \text { reverse }}=-k_{\text {forward }} C_{A}+k_{\text {reverse }} C_{B} C_{C}
$$

These equations allow the full derivation of the rate law for each chemical species involved in the reaction network. If a chemical species is reacting it is given a negative rate, if a chemical species is being produced it is given a positive rate. All chemical reactions are parallel, meaning they occur simultaneously in the model. In order to get the full reaction rate law, all respective individual reaction rate laws were combined to form the overall reaction rate law. For an individual chemical species (i), this can be given from the following equation. [6]

$$
-r_{i, \text { total }}=\sum r_{i, \text { forawrd }}+\sum r_{i, \text { reverse }}=\sum k_{\text {forward }} C_{i} \ldots+\sum k_{\text {reverse }} C_{i} \ldots
$$

The next factor to consider is the type of reactor in which the reaction is taking place. Different types of reactors have different expressions to solve for parameters such as conversion, volume, geometry. For the purpose of this model a plug flow reactor (PFR) or tubular flow reactor was chosen. A plug flow reactor is simply a long straight cylinder 
in which the fluid is in plug flow as shown in Figure 1 [7]. An assumption of plug flow model used here is that there are no temperature, concentration, or reaction rate gradients in the radial dimension. [6] Another characteristic of plug flow is that there is assumed to be no backflow, or back mixing of the fluid. The fluid travels axially down the reactor and exits at the end. Due to the assumption of plug flow, the plug flow reactor can be considered analogous to a batch reactor on some small differential length, $\mathrm{dz}$. This means that the flow traveling down the reactor is only reacting with the same volume of fluid that it entered with. This allows the use of the batch reactor equations to solve for the concentration down the length of the reactor.

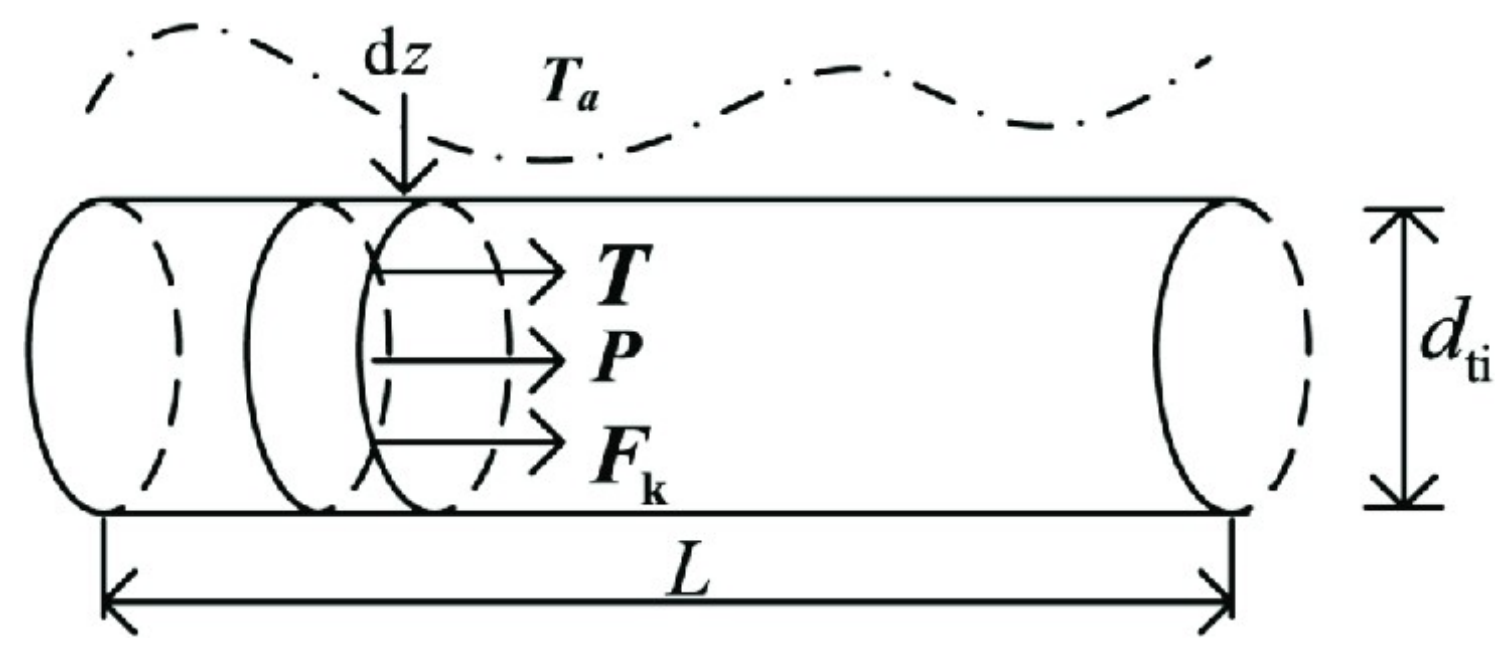

Figure 1. Schematic of a plug flow reactor [7]

The equation for concentration of some chemical species (i) along the length of the reactor under the assumption of plug flow is given below with cross-sectional area $\left(A_{c}\right)$ and axial velocity $\left(v_{\mathrm{z}}\right)$.

$$
\frac{d C_{a}}{d z}=r_{a}
$$


The resulting differential equation is a linear first order ordinary differential equation (ODE). [8] This equation was used to derive the mass balance equations for each species under the assumption of no diffusion. If one or more of the resulting equations contains a variable used in another equation, the equations are said to be coupled. The result is a system of 18 coupled ODEs for the mass balance of the reaction system. The system of ODEs can be represented as show in figure 2 . In this matrix, $y_{n}$ represents the differential equation which is left hand side of the equation. The right side of the equation is the rate law is represented by $\mathrm{f}_{\mathrm{n}}\left(\mathrm{z}, \mathrm{C}_{\mathrm{i}}, \mathrm{C}_{\mathrm{n}}\right)$.

$$
\left[\begin{array}{l}
y_{1} \\
y_{2} \\
y_{3} \\
y_{4} \\
y_{5} \\
y_{n}
\end{array}\right]=\left[\begin{array}{l}
f_{1}\left(z, C_{i}, C_{n}\right) \\
f_{2}\left(z, C_{i}, C_{n}\right) \\
f_{3}\left(z, C_{i}, C_{n}\right) \\
f_{4}\left(z, C_{i}, C_{n}\right) \\
f_{5}\left(z, C_{i}, C_{n}\right) \\
f_{n}\left(z, C_{i}, C_{n}\right)
\end{array}\right]
$$

Figure 2. System of ODEs in matrix form

For most cases using the simple mass balance is acceptable due to the assumption of plug flow. However, there cases when a more complete mass balance can be useful. One such case is when diffusion plays a role in the overall system. To derive the more complex mass balance I turn to "The Equation of continuity for species (a) in terms of concentration (c) for constant $c^{*} D_{a}$ " in cylindrical coordinates. [9]

$$
c\left[v_{r} \frac{d C_{a}}{d r}+v_{z} \frac{d C_{a}}{d z}+\frac{v_{\Theta}}{r} \frac{d C_{a}}{d \Theta}\right]=c D_{a b}\left[\frac{1}{r} \frac{d}{d r}\left(r \frac{d C_{a}}{d r}\right)+\frac{d^{2} C_{a}}{d z^{2}}+\frac{1}{r^{2}} \frac{d^{2} C_{a}}{d \Theta^{2}}\right]+r_{a}
$$


In order to properly use this equation reasonable assumptions, have to be made. The three following assumption were made to end up with the proper mass transfer equation.

1. Incompressible fluid

2. Steady State

3. One dimensional transport (z-dimension)

After applying the assumptions to the equation of continuity, the following equation is derived.

$$
\left[\frac{d^{2} C_{a}}{d z^{2}}\right]=\left(\frac{v_{z}}{D_{a}}\right)\left[\frac{d C_{a}}{d z}\right]-\left(\frac{r_{a}}{c D_{a}}\right)
$$

The resulting equation is the steady-state version of the well-known advection-diffusion equation. [10] This equation requires the calculation of the diffusion coefficient $\left(D_{a}\right)$, axial velocity $\left(v_{z}\right)$, and concentration (c). Most calculations involved in this project did not include the resulting advection-diffusion analog equation. However, a secondary model was eventually built to include the advection-diffusion for mass transport. Including this equation causes a significant increase in computational time. If plug flow and no diffusion are assumed the following equation is used for the mass balance.

$$
\frac{d C_{a}}{d z}=r_{a}
$$


The next section of the model is the energy equation and corresponding heat transfer equations. The general procedure is very similar to the process for the mass transport equations. The first difference between the two is that a simple equation like the first mass transport equation does not exist for a temperature calculation. Due to this it's necessary to start with "The equation of energy for a pure Newtonian fluid" in cylindrical coordinates. [9] The necessary values to calculate are density $\left(\rho_{\mathrm{i}}\right)$, heat capacity $\left(\mathrm{C}_{\mathrm{pi}}\right)$, axial velocity $\left(v_{z}\right)$, thermal conductivity $\left(\mathrm{k}_{\mathrm{mix}}\right)$, and overall heat generation/consumption term $\left(\mathrm{q}_{\mathrm{v}}\right)$. Assumptions must be made to derive the proper equation.

$$
\rho_{i} C_{p i}\left[v_{r} \frac{d T}{d r}+v_{z} \frac{d T}{d z}+\frac{v_{\Theta}}{r} \frac{d T}{d \theta}\right]=k_{m i x}\left[\frac{1}{r} \frac{d}{d r}\left(r \frac{d T}{d r}\right)+\frac{d^{2} T}{d z^{2}}+\frac{1}{r^{2}} \frac{d^{2} T}{d \theta^{2}}\right]+q_{v}
$$

Once the assumptions are properly made the resulting derived energy equation is:

$$
\left[k_{m i x} \frac{d^{2} T}{d z^{2}}\right]=\sum \rho_{i} C_{p i}\left[v_{z} \frac{d T}{d z}\right]-q_{v}
$$

While the resulting energy equation is similar to the advection-diffusion equation derived for the mass balance, the overall heat generation/consumption term must be derived itself. This term represents the overall amount of heat leaving and/or entering the system. For this calculation, the heat being generated/consumed by the gas feed, and heat entering or leaving the system were taken into account. Heat transfer due to radiation was ignored. That leaves two main parts for the evaluation of the heat generation/consumption term. 
To derive the equation for the heat entering the system, it is necessary to figure out how heat could be generated or consumed internally within a system. As previously mention, the EDC cracking process is an endothermic process, so it requires an amount of heat per mol reacted. Only the mass balance for EDC will affect the energy transfer, no other reaction produces or requires heat to execute. This will then be multiplied by the heat of reaction to get the total amount of heat consumed within the system, as is shown below.

$$
\text { Internal heat consumption }=\mathrm{Q}_{\mathrm{con}}=\left(\Delta \mathrm{H}_{\mathrm{rxn}}\right)\left(\sum \mathrm{r}_{E D C}\right)
$$

In order to obtain an expression for the amount of heat leaving/entering the system, certain parameters, geometries, and process assumptions must be made. The differences in the choices made can result in some significantly different expression for the heat leaving/entering the system. This model attempted to choose the most reasonable assumptions and process conditions for the overall reaction system. [11] In most industrial practices, there is some type of heating mechanism to heat the gas feed to designed operating conditions. For the purposes of this model, the temperature of the outside wall was assumed to be held constant, by an outside heating fluid. Due to the assumption of one-dimensional transport equations, heat conduction occurring in the radial direction through the pipe was ignored. This leaves only thermal convection as the method of transferring into the system. In terms of the heat transfer area $(a)$, the overall heat transfer coefficient $\left(\mathrm{U}_{\text {coeff }}\right)$, and (Twall) the temperature of the outside of 
the pipe, which is assumed to be constant. The general equation for thermal convection in a plug flow reactor is as follows:

$$
Q_{\text {in }}=U_{\text {coeff }} a\left(T_{\text {wall }}-T\right)
$$

It should be noted that while this process condition was chosen for this model, other methods such concurrent or counter-current cooling/heating jackets are often commonly used. [12] the equations for internal heat consumption and external heat loss can now be plugged into the simplified energy balance to obtain the final energy balance equation.

$$
\begin{gathered}
\mathrm{q}_{\mathrm{v}}=\mathrm{U}_{\text {coeff }} a\left(\mathrm{~T}_{\text {wall }}-\mathrm{T}\right)+\Delta \mathrm{H}_{\mathrm{rxn}} \sum r_{E D C} \\
{\left[k_{\text {mix }} \frac{d^{2} T}{d z^{2}}\right]=\sum \rho_{i} C_{p i}\left[v_{0} \frac{d T}{d z}\right]-\left[\mathrm{U}_{\text {coeff }} a\left(\mathrm{~T}_{\text {wall }}-\mathrm{T}\right)+\Delta \mathrm{H}_{\mathrm{rxn}} \sum r_{E D C}\right]}
\end{gathered}
$$

Every necessary mass and balance equation have now been successfully derived.

To successfully set up and evaluate the system of ODEs however, it is necessary to calculate many different constants for both the mass and energy balances. Under normal circumstances most of these parameters would be sufficiently easy to calculate and use. Unfortunately, the complexity of the ODE system and mixture of chemicals requires some more rigorous and challenging calculations.

First are the parameters for the mass balance. In total there are three necessary parameters that need to be calculated, axial velocity $\left(v_{z}\right)$, concentration (c), and 
diffusion coefficient $\left(D_{a}\right)$. To calculate axial velocity values for initial volumetric flow $(\dot{V})$, and inner radius of the pipe will need to be provided. Values of $1.5 \mathrm{~m}^{3} / \mathrm{s}$ and $0.225 \mathrm{~m}$ were used for volumetric flow and inner radius respectively. Concentration is simply the summation of all individual concentrations for each individual species in the gas mixture.

$$
\begin{gathered}
a=\frac{2 \pi r_{i} L_{i}}{\pi r_{i}^{2} L_{i}}=\frac{2}{r_{i}} \\
c=\sum C_{i}
\end{gathered}
$$

The calculation of the diffusion coefficient is more complicated than the other mass balance parameters. In the process of calculating the diffusion coefficient, the Python library thermo [13] was used to calculate intermediary values needed. This was in part due to the complex nature of the calculations for large gas mixtures. [14] An approximate formula for a binary diffusion coefficient is give below [15], where $\left(\mu_{a}\right.$, $\left.\mu_{b}\right)$ are the molecular masses, $(P)$ is the pressure of the gas mixture, $(\mathrm{T})$ is the temperature of the gas mixture, $\left(\sigma_{\mathrm{ab}}\right)$ is the interaction parameter, and $\Omega$ is the collision integral [16].

$$
D_{a b}=\frac{1.8583 * 10^{-5} T^{3 / 2}\left(\frac{1}{\mu_{a}}+\frac{1}{\mu_{b}}\right)^{1 / 2}}{P \sigma_{a b}^{2} \Omega}
$$




$$
\frac{\varepsilon_{a b}}{k}=\left(\frac{\varepsilon_{a}}{k} \frac{\varepsilon_{b}}{k}\right)^{1 / 2}, \sigma_{a b}=\frac{\sigma_{a}+\sigma_{b}}{2}
$$

Once every binary diffusion coefficient has been calculated, the effective diffusion coefficient of chemical a $\left(D_{a}\right)$ in the gas mixture can be calculated from the following relationship using the molar fractions $\left(Y_{a}\right)$ and binary diffusion coefficients $\left(D_{a b}\right)$ of the gas mixture. $[15,16]$

$$
D_{a}=\frac{1-Y_{a}}{\frac{Y_{b}}{D_{a b}}+\frac{Y_{c}}{D_{a c}}+\ldots \frac{Y_{n}}{D_{a n}}}
$$

In the process of calculating the individual diffusion coefficient, thermo was used to calculate the interaction parameters and the collision integral [17]. Once all necessary individual diffusion coefficients were calculated all required parameters for the mass balance have been calculated.

The last necessary parameters required before the completion of the model are the energy equation parameters. These parameters include, component density $\left(\rho_{i}\right)$, component heat capacity $\left(C_{p i}\right)$, thermal conductivity of the gas mixture $\left(k_{m i x}\right)$, and axial velocity $\left(v_{z}\right)$. If individual component gas densities are not known they can be calculated using the following correlation:

$$
\rho_{i}=\frac{P_{i}}{R T}
$$


Calculating the values for $C_{p i}$ involves using the Lastovka Shaw method [18], this property was calculated using the thermo package. The thermal conductivity of the gas mixture was calculated using the method described in [18] [19] shown below.

$$
\begin{gathered}
k=\sum_{i=1}^{n} \frac{y_{i} k_{i}}{\frac{1}{y_{i}} \sum_{j=1}^{n} A_{i j} y_{j}} \\
A_{i j}=\frac{1}{4}\left\{1+\left[\frac{\eta_{i}}{\eta_{j}}\left(\frac{M W_{j}}{M W_{i}}\right)^{0.75}\left(\frac{T+S_{i}}{T+S_{j}}\right)\right]^{0.5}\right\}^{2}\left(\frac{T+S_{i j}}{T+S_{i}}\right) \\
S_{i j}=0.733\left(S_{i} S_{j}\right)^{0.5} \\
S_{i}=1.5 T_{B}
\end{gathered}
$$

Where Temperature $(T)$, mole fraction $\left(y_{i}\right)$, and boiling temperature $\left(T_{B}\right)$ are the necessary inputs. The overall heat transfer coefficient can be calculated using the following correlation: [20]

$$
\begin{gathered}
U=\frac{1}{\left[\left(\frac{d_{o}}{d_{i}}\right)\left(\frac{1}{h_{i}}\right)+\left(\frac{d_{o} * \ln \left(\frac{d_{o}}{d_{i}}\right)}{2 k_{\text {pipe }}}\right)+\left(\frac{1}{h_{o}}\right)\right]} \\
h=\frac{k N_{u}}{L}
\end{gathered}
$$

The Nusselt number has many different correlations but since flow is almost always turbulent the following method is used most. [21] 


$$
\begin{gathered}
N_{u}=0.027 R_{e}^{(4 / 5)} P_{r}^{(1 / 3)}\left(\frac{\mu}{\mu_{w}}\right)^{0.14} \\
R_{e}=\frac{\rho v_{z} L}{\mu} \\
P_{r}=\frac{C_{p} \mu}{k}
\end{gathered}
$$

Finally, the viscosity of the gas mixture $(\mu)$ and the viscosity of the gas mixture at the wall $\left(\mu_{w}\right)$ were calculated using the equations from [22].

$$
\begin{gathered}
\eta_{m i x}=\sum_{i=1}^{n} \frac{y_{i} \eta_{i}}{\sum_{j=1}^{n} y_{j} \phi_{i j}} \\
p h i_{i j}=\left(\frac{\eta_{i}}{\eta_{j}}\right)^{0.5} S_{i j} A_{i j} \\
A_{i j}=m_{i j} M_{i j}^{-0.5}\left[\begin{array}{l}
2\left(1+M_{i j}\right)+\frac{\left(1+M_{i j}^{0.45}\right) m_{i j}^{-0.5}}{1+m_{i j}} \\
M_{i j}=\left[\frac{4}{\left(1+M_{i j}^{-1}\right)\left(1+M_{i j}\right)}\right]^{0.25} \\
S_{i j}=\frac{M_{i j}=\frac{M_{i}}{M_{j}}}{\left.11+T_{i}^{*}+\left(\delta_{i}^{2} / 4\right)\right]^{0.5}\left[1+T_{j}^{*}+\left(\delta_{j}^{2} / 4\right)\right]^{0.5}}
\end{array}\right.
\end{gathered}
$$




$$
T^{*}=\frac{k T}{\varepsilon}
$$

Now that every necessary parameter has been determined both the energy and mass balances are ready to evaluate. However, the solver that this model used to numerical integrate the system of ODEs requires that every equation be a first order differential equation. This means that the energy and mass balance equations must be transformed into first order from second order. Any liner nth order differential equation can be reduced into a system of liner ODEs through the following method.

$$
\begin{gathered}
\mathrm{a}_{2} \mathrm{y}^{\prime \prime}+\mathrm{a}_{1} \mathrm{y}^{\prime}+\mathrm{a}_{0} \mathrm{y}=0 \\
x_{1}=\mathrm{y}, x_{2}=\mathrm{y}^{\prime}=x^{\prime}{ }_{1}, x^{\prime}{ }_{2}=\mathrm{y}^{\prime \prime} \\
\mathrm{x}_{1}^{\prime}=\mathrm{x}_{2} \\
x_{2}^{\prime}=-\frac{a 1}{\mathrm{a} 2} \mathrm{y}^{\prime}-\frac{a 0}{\mathrm{a} 2} \mathrm{y}=-\frac{a 1}{\mathrm{a} 2} x_{2}-\frac{a 0}{\mathrm{a} 2} x_{1}
\end{gathered}
$$

Applying the process to the second order mass balance gives the following equation.

$$
\begin{aligned}
& x_{1}=\mathrm{C}_{\mathrm{a}} \\
& x_{2}=\frac{\mathrm{dC}_{\mathrm{a}}}{\mathrm{dz}}=x^{\prime}{ }_{1} \\
& x^{\prime}{ }_{1}=x_{2} \\
& x^{\prime}{ }_{2}=\left(\frac{v_{\mathrm{z}}}{\mathrm{D}_{\mathrm{a}}}\right) x_{2}-\left(\frac{r_{\boldsymbol{a}}}{c D_{a}}\right)
\end{aligned}
$$


Once this has been complete for every single chemical species, there are a total of 36 mass balance equations that must be put into the ODE system. Within each pair of mass balances, $x_{1}^{\prime}$

Would be considered the solution for the desired chemical species i.e. (EDC, $\mathrm{HCl}, \mathrm{VCM}$ ) and $x_{2}^{\prime}$ would be directly coupled and required to solve to obtain an answer for the desired chemical species. This same procedure applied then to the energy equation would result in the following:

$k_{1}=\mathrm{T}$

$k_{2}=\frac{\mathrm{d} T}{\mathrm{dz}}=k_{1}^{\prime}$

$k^{\prime}{ }_{1}=k_{2}$

$k_{2}^{\prime}=\left(\frac{\boldsymbol{v}_{\boldsymbol{z}} \sum \boldsymbol{\rho}_{i} \boldsymbol{C}_{\boldsymbol{p} i}}{\boldsymbol{k}_{\text {mix }}}\right) k_{2}-\left(\left(\frac{\mathbf{U}_{\text {coeff }} \boldsymbol{a}}{\boldsymbol{k}_{\text {mix }}}\right)\left(\mathrm{T}_{\mathrm{wall}}-k_{1}\right)+\left(\frac{\Delta \boldsymbol{H}_{r x n}}{\boldsymbol{k}_{\text {mix }}}\right) \sum \boldsymbol{R}_{E D C}\right)$

As the ODE system is fully computed and ready to go, it can now be organized into the matrix form displayed in Figure 2. The order doesn't seem to make a difference as long as the correct equations and values are paired. When programming the ODE system, the model worked best when the equations for the Arrhenius equation is directly substituted for k, rather than calculating it before it is entered into the solver.

$$
\mathrm{k}=k_{0} e^{\left(\frac{-E a}{R T}\right)}
$$

This completes the ODE system and fully prepares it to be numerical integrated by the chosen solver. The final section regards the setup and evaluation of the complete ODE system in Python and the solutions to the problems that show up when trying to set it 
up as the documentation suggests. While there are different methods to solve a given initial value problem in Python and its respective libraries, most were unable to handle the problem correctly. The SciPy method "solve_ivp" was the only available solver that could routinely solve the system it was presented with. While there where many times where it either failed to converge, crash, or experiences other numerical issues, it is the only reliable solver for a system this large and complex.

The solve_ivp method requires the following minimum arguments in order to correctly function. The first is "fun" or the function that returns the system of ODEs when being called. "fun" needs to have two input variables, "fun $(t, y)$ " or more specific to our system "fun $(z, C)$ ", args and kargs do not natively work with this method. Without any extra arguments, $t / z$ can just be a Sympy symbol as it won't be used at all during the entire process. Variable $\mathrm{y} / \mathrm{C}$ should generally be a list of Sympy symbols of all the chemical reactants, temperature, and differential terms. When this function is called by solve_ivp it should return a list of all the equations on the right-hand side of the equation. In the absence or extra args, "fun" can just be called without input variables or parentheses i.e. "fun". However, if it is necessary to include any extra arguments of any kind the "fun" input changes entirely. In this scenario fun would have to be called exactly like this in order to function properly, "lambda z, C: $\operatorname{rhs}\left(z, C,{ }^{*} \operatorname{args} 1\right)$ ". This also requires the ODE system to consist entirely of Sympy symbols including the left-hand variable.

The next parameter required is "t_span". This is simply a tuple of starting and stopping points for the solver i.e. " $(0,100)$ ". Next is the input variable " $y 0$ ", which is 
simply a list of all the initial values for each respective variable in the ODE system. The last input parameter isn't technically always required, but it is necessary for this system for function properly. The input parameter is the specific method the solver should use to solve the system of ODEs. There are five in total, "RK45"," RK23"," Radau"," BDF", and "LSODA". The first two methods, "RK45" and" RK23", are for simple systems that aren't stiff. A stiff system of ODEs either takes too long or fails to converge to an answer. Since the system of ODEs that was built is stiff these two methods do not function correctly and will not reach an answer. [23] The next two solvers," Radau" and" BDF", are methods that are more likely to solve stiff sets of ODEs. For this model in particular, "Radau" was the solver used almost exclusively. The last method is "LSODA", which is a method designed to switch between stiff and un-stiff solvers when it detects which behavior is happening at the current iteration. [24]

One last parameter to consider in the Jacobian, which is a matrix of every possible derivative on every equation on the system. The Jacobian is useful to improve the accuracy of the solve_ivp method.

$$
\mathbf{J}=\left[\begin{array}{lll}
\frac{\partial \mathbf{f}}{\partial x_{1}} & \cdots & \frac{\partial \mathbf{f}}{\partial x_{n}}
\end{array}\right]=\left[\begin{array}{ccc}
\frac{\partial f_{1}}{\partial x_{1}} & \cdots & \frac{\partial f_{1}}{\partial x_{n}} \\
\vdots & \ddots & \vdots \\
\frac{\partial f_{m}}{\partial x_{1}} & \cdots & \frac{\partial f_{m}}{\partial x_{n}}
\end{array}\right]
$$

Figure 3. Jacobian of a given system in matrix form

For the solver methods, "RK45" and" RK23", calculating the Jacobian doesn't help in any way as they not designed to accept a Jacobian as an input variable. The other three 
methods can accept it and it is recommended by the documentation to provide one or it will be forced to use the finite-difference approximation. "Radau" and "BDF" generally function the same way in terms of the Jacobian. "LSODA" requires that the returned values or function not be a sparse matrix, which often happens with larger systems.

When symbolically calculating the Jacobian there are numerous ways to get a technically correct answer. It should also be noted that when taking the energy equation into account the symbolically generated exponent terms for the reaction constant does not allow anything except a Sympy symbol for the temperature term and cannot be numerically evaluated for the Jacobian. This can cause problems when attempting to use libraries that extend the numerical range of the program (Decimal, mpmath) etc. This can be solved by creating a separate RHS function for the function called by the method and the function called for the Jacobian. The following is the generic code that functions correctly with the solve_ivp method:



Figure 4. Code used to symbolically calculate the Jacobian

It should also be noted that due to the complex nature of the system, the solver will sometimes fail to converge or get an error saying, "rate was defined before it was 
used". This tends to happen when the numbers in the solver become too small or too large to handle. The only solution to this problem seems to be setting a "good" "first_step". With setting "Ls" equal to the length in the $z$ direction of each interval, the best first_step values were either "(Ls/100.0)" or “(Ls/1000.0)" or simply “1E-3". Anything higher or lower than these numbers will cause the system to potentially fail. For the vast majority of calculations in the program, the module "Decimal" is being used to increase the accuracy beyond normal float values. However, the Decimal objects get internally converted into a normal float by the solve_ivp method. The two necessary arguments for the solver method, the absolute and relative tolerance, were each set to 1E-7. At some point during the evaluation of the programs, one or more likely both solvers will return an overflow error. This is fixed by then changing both tolerances values to $1 \mathrm{E}-3$. Setting the precision lower may cause the solver to fail. Future work could increase the number of intervals and decrease the amount of activation energy changed per iteration. Both the absolute and relative tolerance were set to $1 \mathrm{E}-7$ and changed if an overflow error is returned. These parameters prevented the solving method from diverging towards unrealistic or impossible answers i.e. negative temperatures. The precision for both solvers were set at 100 and had an iterative loop to temporarily increase the precision if necessary. 


\section{Results and Discussion}

Before the solver was run, the following initial conditions were set. Pressure was set to 12 bar or 11.84 atm. Pressure was assumed to be constant throughout the length of the pipe. Initial volumetric flow was set to $1.5 \mathrm{~m}^{3}$. The outer diameter of the pipe was set to $50 \mathrm{~cm}$ or $0.5 \mathrm{~m}$. The thickness of the pipe was set to $2.5 \mathrm{~cm}$ making the inner diameter of the pipe $45 \mathrm{~cm}$ or $0.45 \mathrm{~m}$. When iteratively changing the initial activation energy for the first reaction, initial temperature was set to $500^{\circ} \mathrm{C}\left(773.15^{\circ} \mathrm{K}\right)$. The reaction was to be carried out for a total of 30 seconds. Each iteration subtracted a value of $0.25 \mathrm{~kJ} / \mathrm{mol}$ from the first reaction for a total range of $(342.0-317.0) \mathrm{kJ} / \mathrm{mol}$. When iteratively changing the initial temperature, the initial temperature was set to 650 ${ }^{\circ} \mathrm{C}\left(923.15^{\circ} \mathrm{K}\right)$. Each iteration subtracted a value of $1{ }^{\circ} \mathrm{C}(1 \mathrm{~K})$ and was performed 200 times for a total range of $(650-450)^{\circ} \mathrm{C}$.

The length of the reactor was split into segments, with each iteration integrating over the length of the reactor segment. The segment number was set at 10 segments a second, for a total of 300 segments. Three variations of the solver were used. The first does not include either diffusion or a Jacobian. The second includes a Jacobian but does not include diffusion. The final variation includes both diffusion and the Jacobian. Unless otherwise specified, the results shown were obtained from the solver utilizing a Jacobian but ignoring diffusion. 
At initial conditions for both activation energy and temperature, the overall conversion of EDC is shown below with a final conversion of approximately $52 \%$. The difference between this and the values obtained by other literature [3], by $8 \%$ given the same exact initial conditions. This is thought to be due to the exclusion of $\mathrm{CCl}_{4}$ as early solver builds obtained similar values as [3] with the inclusion of $0.5-2.0 \% \mathrm{CCl}_{4}$ by composition.

The first variable that was experimented with was activation energy $\left(E_{a}\right)$. Activation energy plays a substantial role in determining reaction kinetics. The beginning reaction has a significantly higher activation energy $(342.0 \mathrm{~kJ} / \mathrm{mol})$ that all other reactions. This results in the reaction being limiting as all other reactions depend upon it either directly or indirectly.

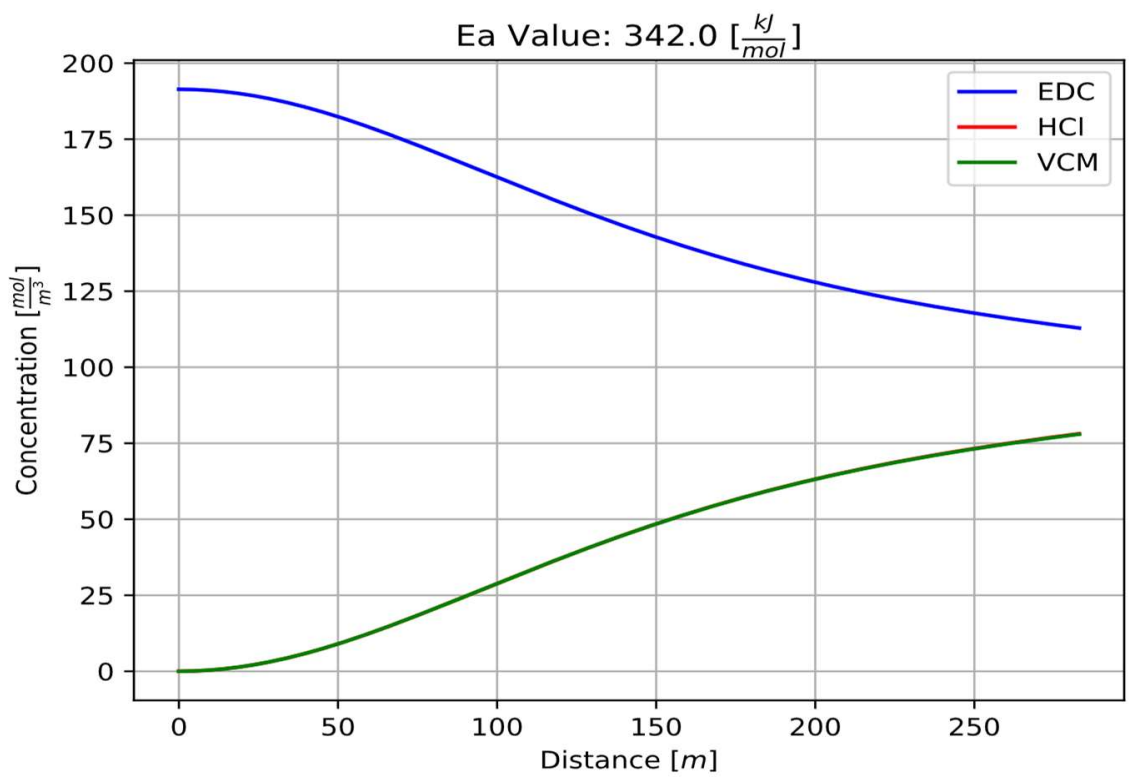

Figure 5. Concentration profile of $E D C, H C l$, and VCM at the initial operating conditions

Decreasing the limiting reactions activation energy by small amounts over the selected range demonstrates its role in the process and its effect on the overall outcome 
of the reaction process. However, if the activation energy was set too low, the solver would either fail or produce results to close to $99-100 \%$ to be considered stable. For this reason, an amount of 250 joules $(0.25 \mathrm{~kJ})$ per mol was reduced each iteration to prevent numerical failures and clearly demonstrate the effect of activation energy on the overall system. Since the overall process is endothermic, it requires heat input to convert the starting material. Lowering the activation energy directly lowers the required heat input and thus increases the reaction rate. Due to the complexity of the system, the effects of activation energy on the purity of the products had to be determined experimentally through the use of the model. Product purity is considered to be the ratio of the desired products ( $\mathrm{HCl}$ and $\mathrm{VCM})$ to the total amount of produced substances excluding EDC.

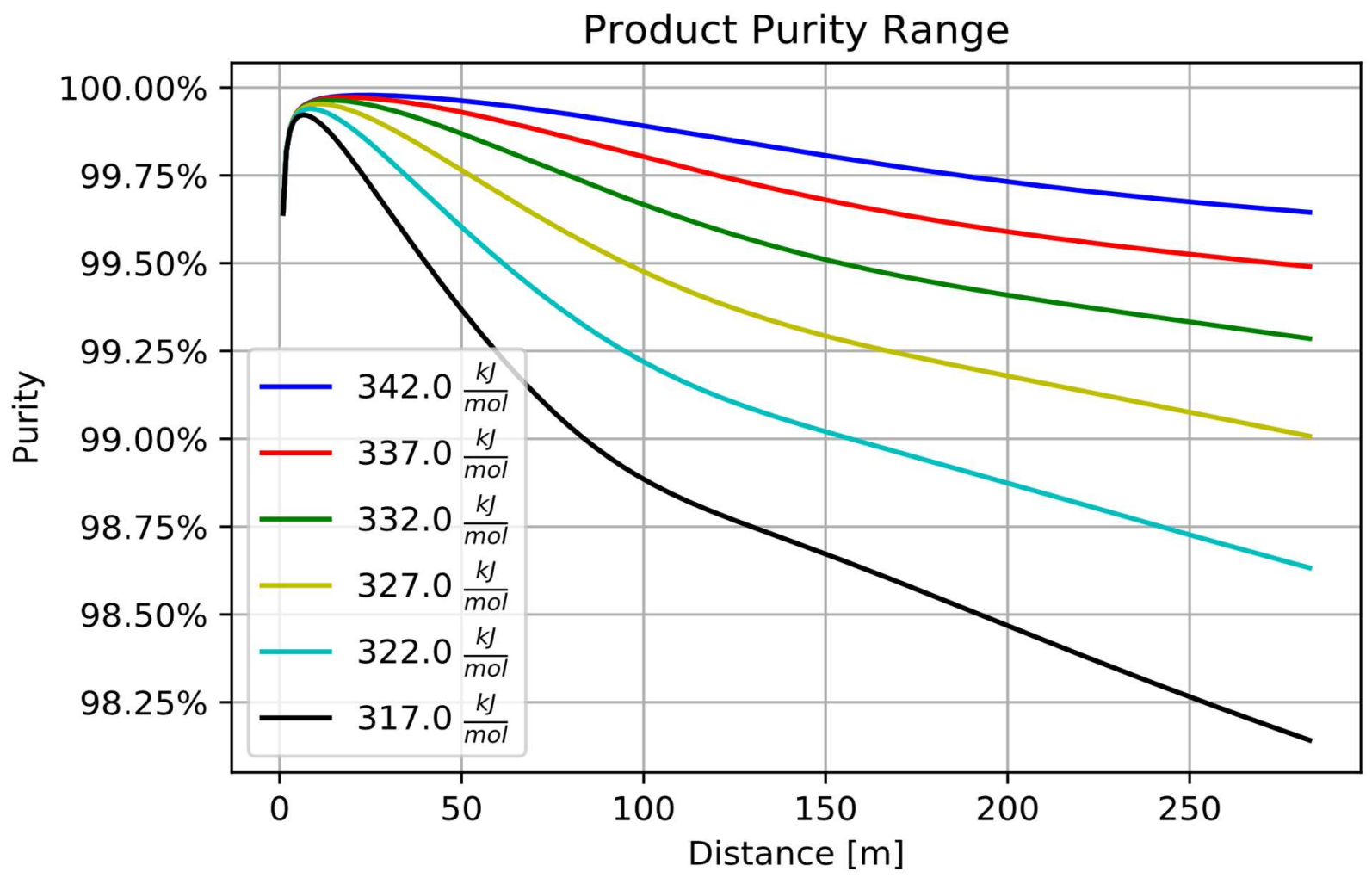

Figure 6. Product Purity over a range of initial Ea Values 


$$
\begin{gathered}
\boldsymbol{C}_{\text {products }}=C_{\text {total }}-C_{E D C} \\
\boldsymbol{C}_{\text {desired }}=C_{H C l}+C_{V C M} \\
\text { Purity }=\frac{C_{\text {desired }}}{C_{\text {products }}+C_{\text {desired }}}=\frac{C_{\text {desired }}}{C_{\text {total }}-C_{E D C}}
\end{gathered}
$$

As shown in the figure above, the purity of the gas mixture is not significantly affected by decreasing the limiting reactions activation energy, maintain approximately $98 \%$ purity at $317.0 \mathrm{~kJ} / \mathrm{mol}$, its lowest value. The results demonstrate the retention of a very high purity which is a crucial condition of the operating conditions, over the entire range of tested values.



Figure 7. Overall Conversion of EDC 
Decreasing activation energy has a directly inversely proportional effect on conversion. The lower its decreased, the higher conversion is obtained. It was also shown that both solvers, without or without a Jacobian, obtained very similar values for their respective final conversion. A decrease of $10.0-15.0 \mathrm{~kJ}$ per mol reacted would increase the conversion of EDC to $75-80 \%$. This offers a significant increase in conversion for a very reasonable decrease in activation energy, while also keeping product purity in the desired range. As the solver got closer to the final activation energy value, the system got increasingly unstable with respect to its numerical integration. The solvers repeatedly ran into numerical issues and failed shortly after a value of $317.0 \mathrm{~kJ} / \mathrm{mol}$, ending on a conversion of approximately $95 \%$. However, since the conversion is

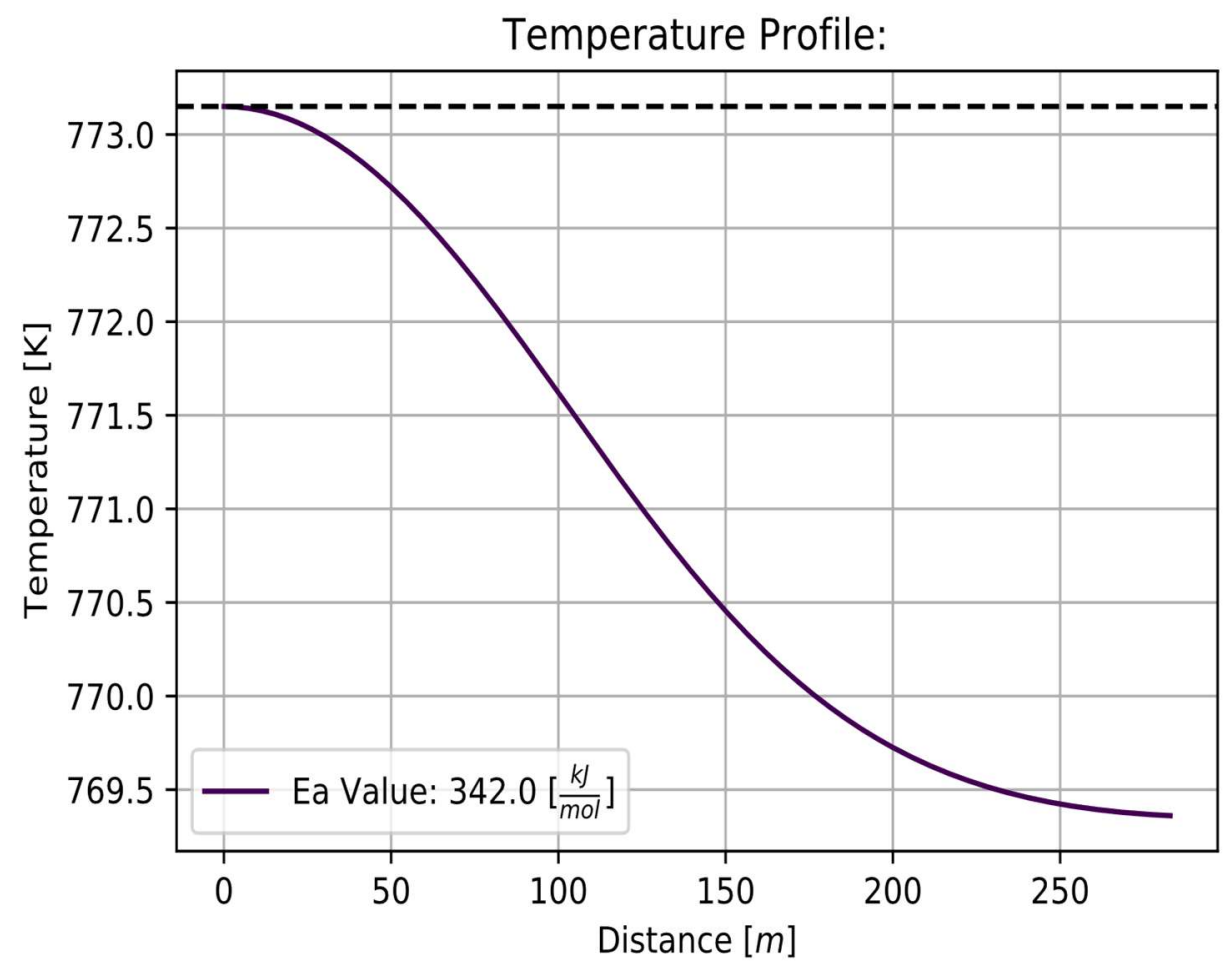

Figure 8. Temperature profile of the gas mixture along the reactor 
approaching $100 \%$ near this value, it is suspected that this is the source of the instability.

In terms of the energy balance, the outside temperature of the wall was assumened to be constant. The energy consumend by the reaction is replaced with heat from the outer wall, since this energy input is constant the temperature of the gas changes axially. The temperature of the outer wall is set to be exactly the same as the temperature of the initial gas temperature. The rate of energy consumption rapidly increases as the limiting reaction rate grows exponentially. Once the majority of the EDC has reacted the temperature starts to regain stability and returns back to normal operating conditions. If the reaction were to continue on, the outer wall temperature would eventually return to its initial condition. The overall change in temperature is 7-9 K, so appears to not be significant.

Each increment of $5.0 \mathrm{~kJ}$ per mol produces significant increases in conversion while mainting product purity. This greatly incentivies research into a possible catalyst for this process as the improvement in the conversion by an amount of $10 \%$ or greater would save time, money, and reduce unwanted by-products. The conversion may vary depending on the choice of operating conditions and initial values, such as temperature, pressure, volumetric flow rate, and time. However, the proportion of increase by lowering the activation energy remains the same or very similar regardless of starting conditions. 




Figure 10. Concentration profile of EDC, $\mathrm{HCl}$, and VCM with an initial Ea of 337.0

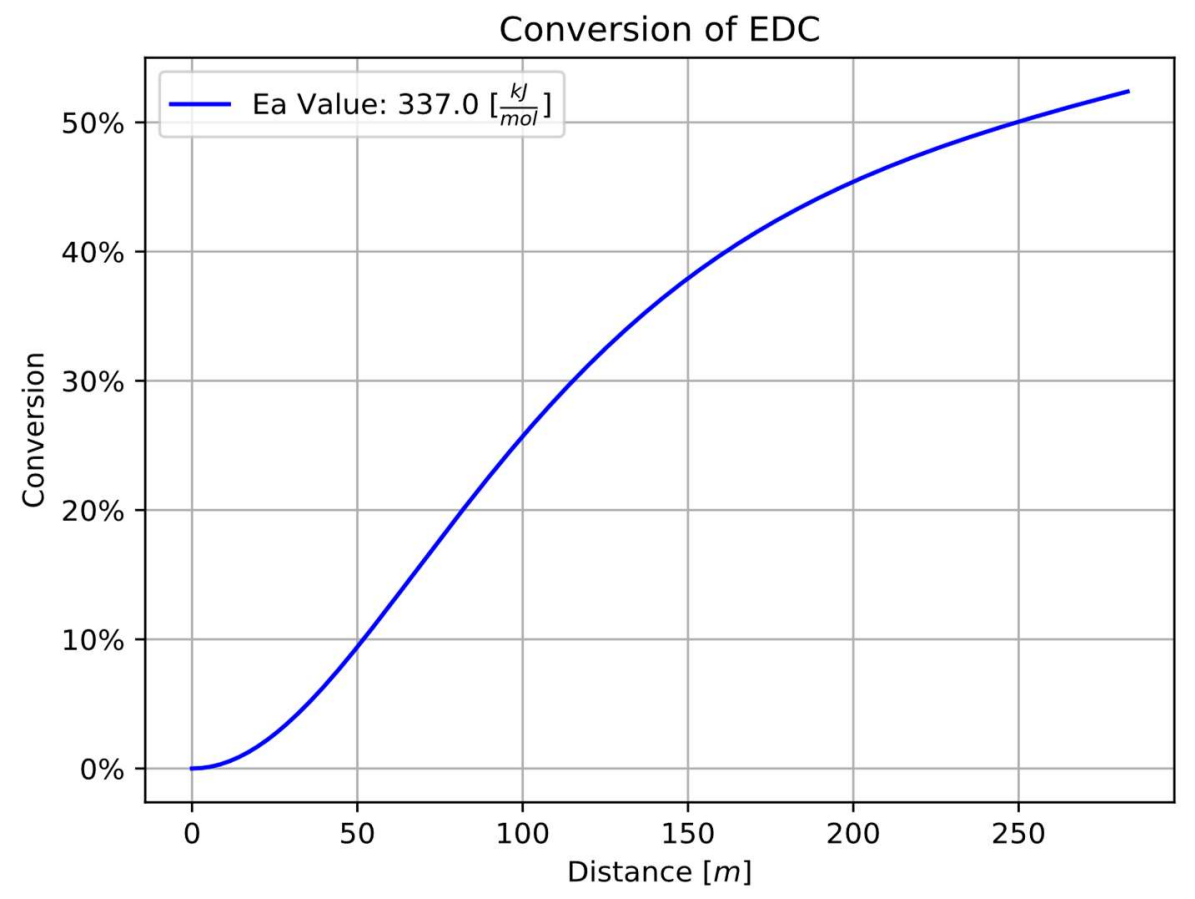

Figure 9. Conversion profile of EDC at an Ea value of 337.0 


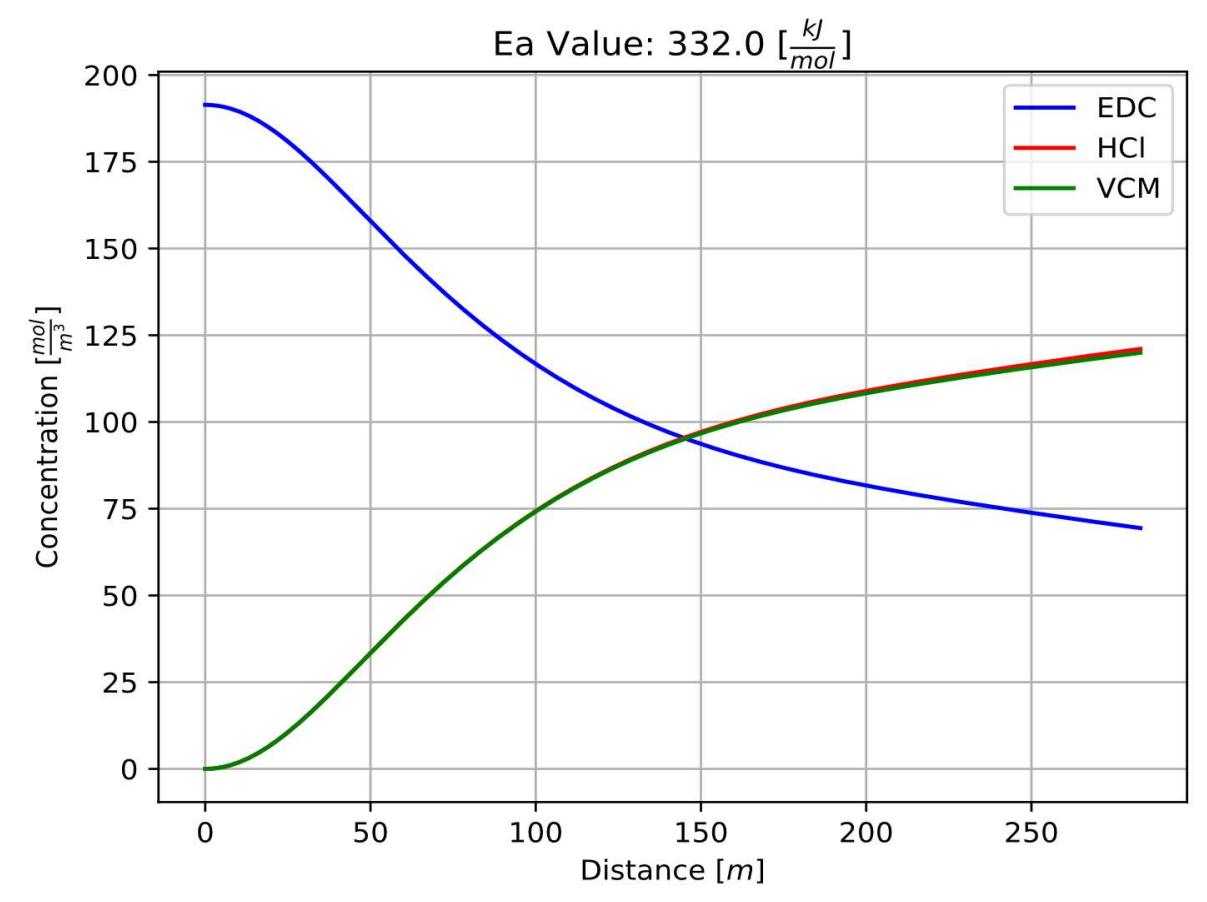

Figure 12. Concentration profile of $E D C, H C l$, and VCM with an initial Ea of 332.0

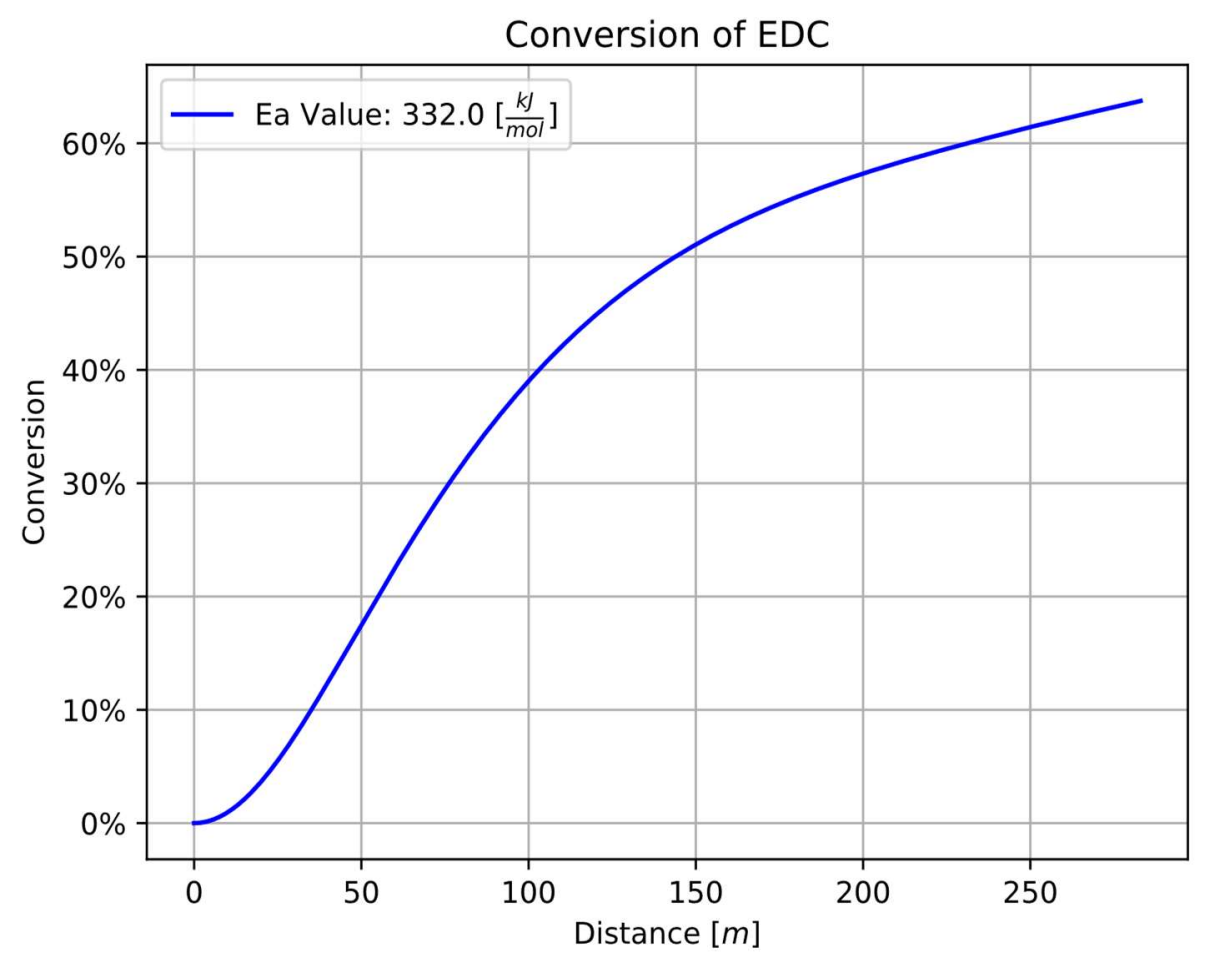

Figure 11. Conversion profile of EDC at an Ea value of 332.0 
When run at the initial operating conditions, the conversion only reaches $41 \%$. Under the same conditions, improvements in conversion were observed similar to those under the normal operating conditions. For every $5.0 \mathrm{~kJ}$, the conversion increased by approximately $10 \%$.

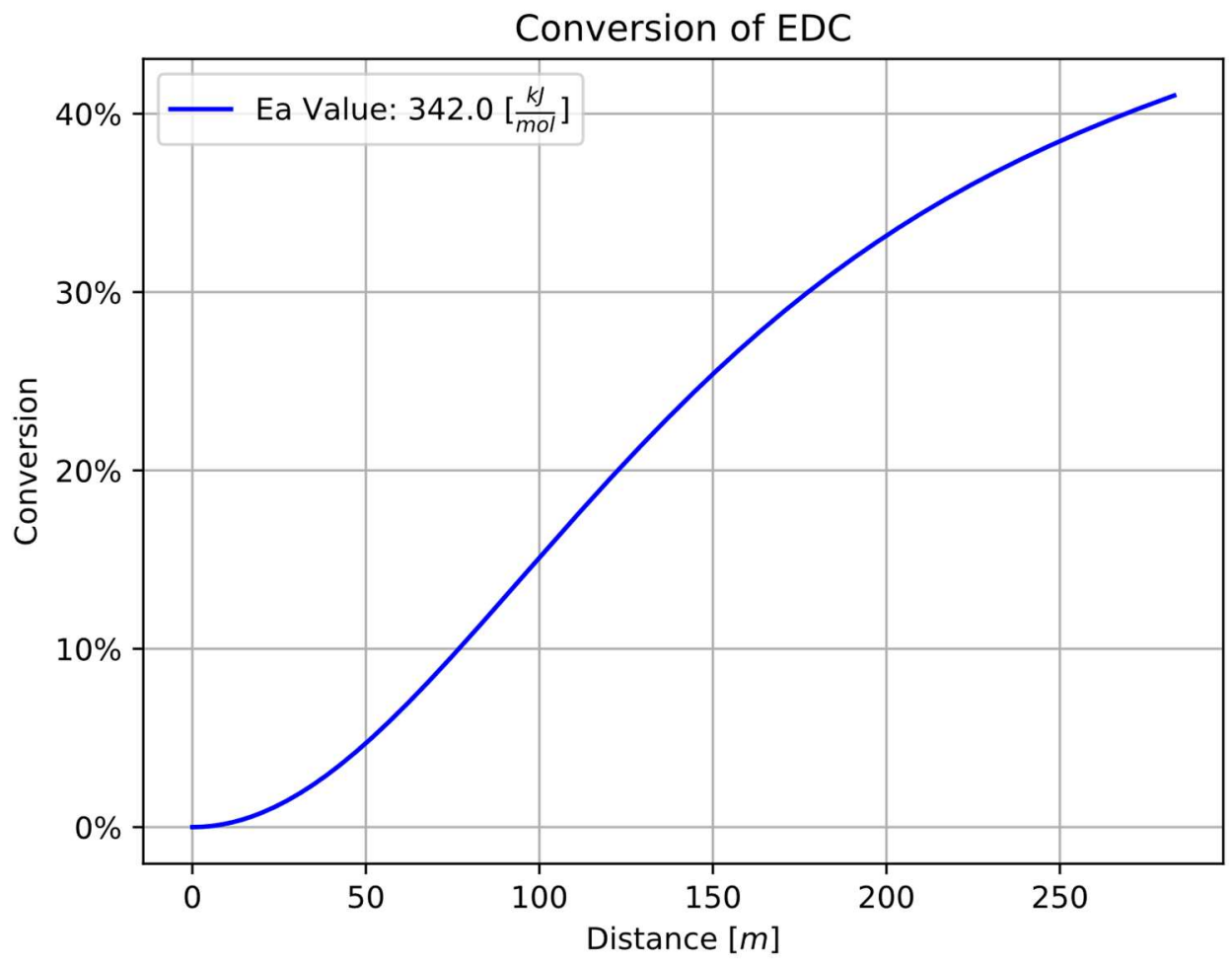

Figure 13. Conversion profile of EDC at an Ea value of 342.0

In terms of the energy balance, there are three constants that are evaluated at each interval. Constant 1 is the differential term coefficient $\left(\frac{d T}{d z}\right)$, Constant 2 is the convection coefficient $\left(T_{\text {wall }}-T\right)$, and Constant 3 is the heat generation/loss coefficient. These values are normally considered to be constant, but through the use of many different thermo modules, they were iteratively calculated. These constants can vary significantly in some cases so attention to their variation can give insight into how 


\section{Constant 1}

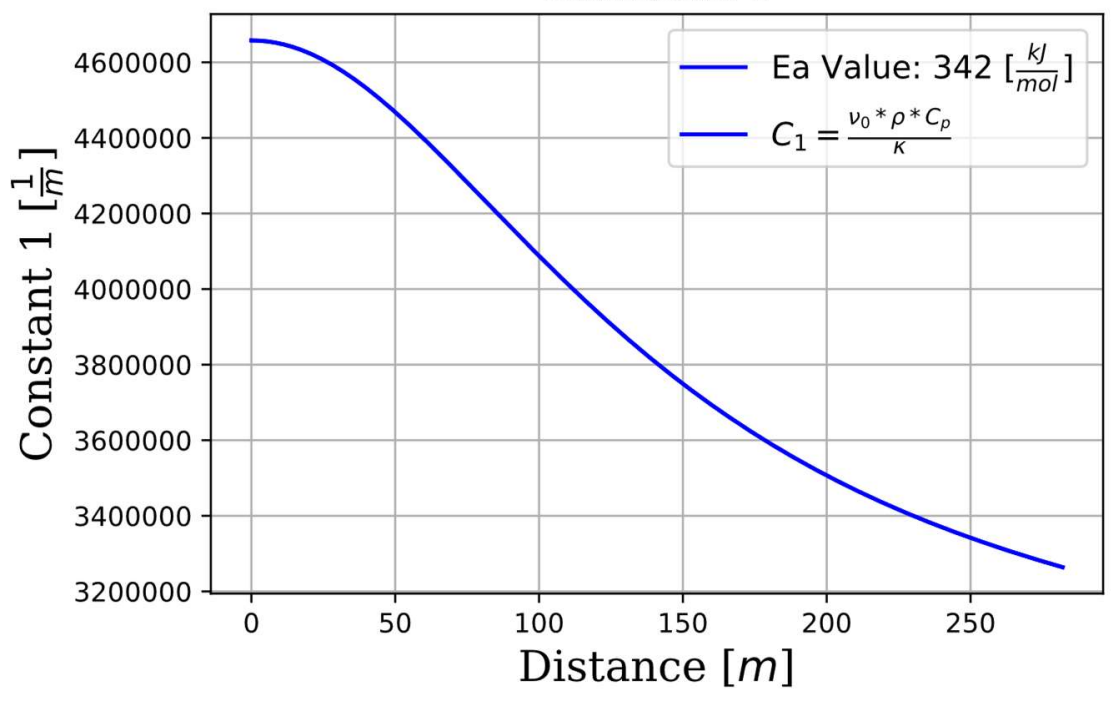

Figure 14. Constant 1 Profile, calculated using the equation shown in the above figure

constant they actually turn out. For Constant 1 the terms in the equation are initial axial velocity $v_{0}\left(\frac{m}{s}\right)$, density of the gas mixture $\rho_{0}\left(\frac{m o l}{m^{3}}\right)$, constant pressure heat capacity of the gas mixture $C_{p}\left(\frac{J}{m o l ~ K}\right)$, and thermal conductivity of the gas mixture $\mathrm{k}\left(\frac{W}{m K}\right)$. As the composition of the gas mixture changes, the thermodynamic properties and transport

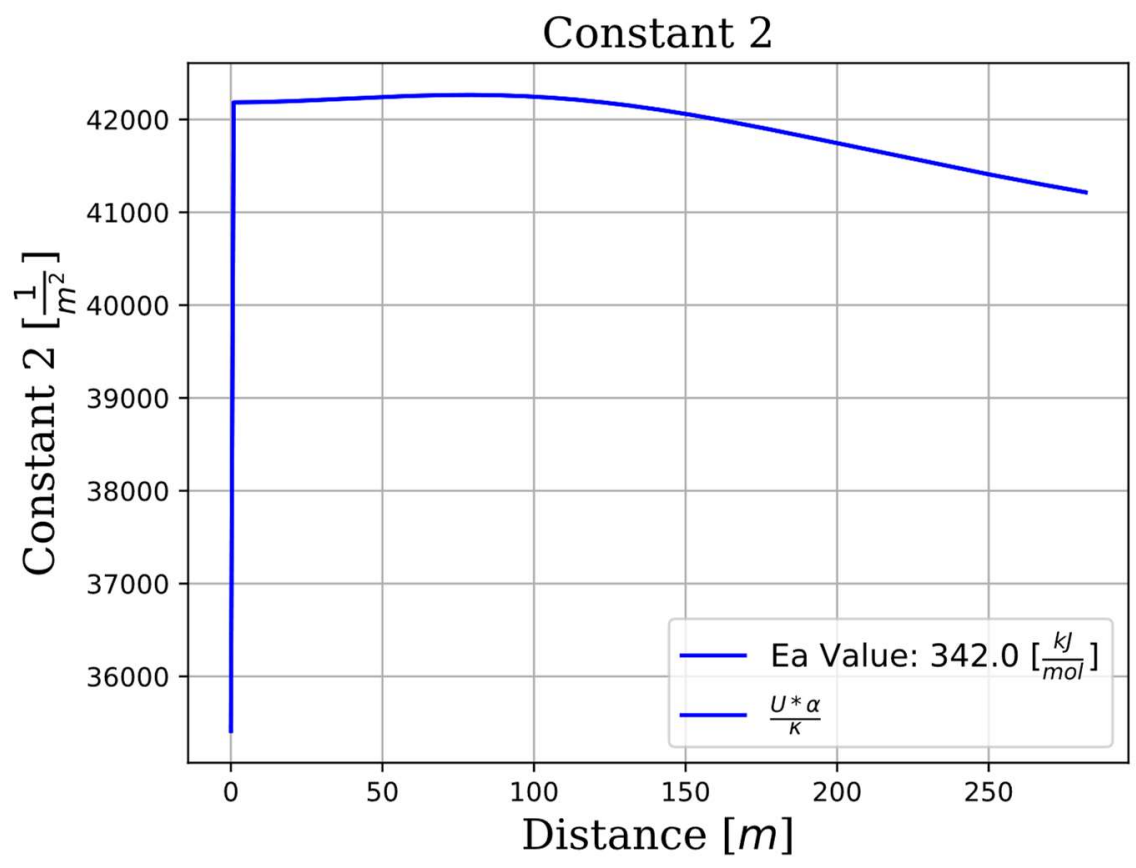

Figure 15. Constant 2 Profile, calculated using the equation shown in the above figure 
coefficients will change with it. This in turn affects the constant coefficients. As shown above, this can sometimes be quite significant. Constant 2 has the following parameters, alpha $a\left(\frac{1}{m}\right)$, overall heat transfer coefficient $U\left(\frac{W}{m^{2} * K}\right)$, and thermal conductivity of the

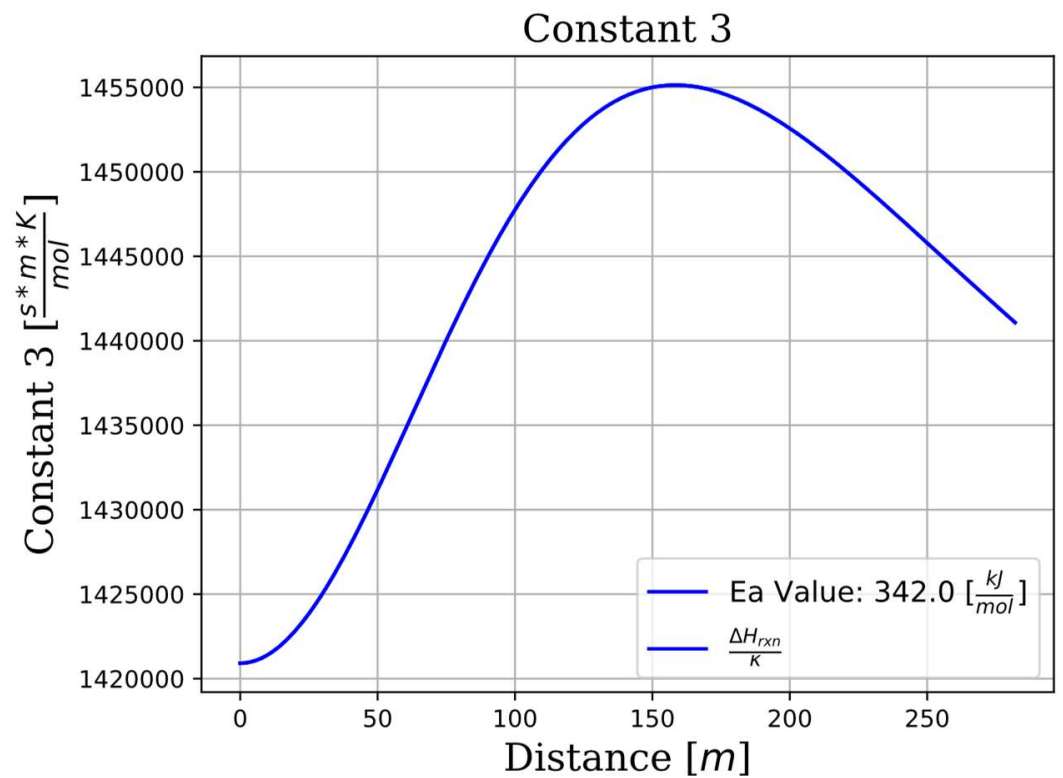

Figure 16. Constant 3 Profile, calculated using the equation shown in the above figure

gas mixture $\mathrm{k}\left(\frac{W}{m K}\right)$. Constant 3 has the following two parameters, heat of reaction $\Delta H_{r x n}\left(\frac{J}{m o l}\right)$, and thermal conductivity of the gas mixture $\mathrm{k}\left(\frac{\mathrm{W}}{\mathrm{mK}}\right)$.

Constant 2 was much steadier overall compared to Constant 1 and Constant 3. The initial spike seen in the beginning is due to the very first calculated value being determined from the first reaction products and EDC. This is likely due to the fact that temperature changes are small and will have less of an effect on the overall system. It also must be considered that half of the convection equation ( $\left.T_{\text {wall }}\right)$ is held constant. Both the graph of the overall heat transfer coefficient $(U)$ and heat transfer coefficient (h) follow an extremely similar pattern to the graph of Constant 2. Constant 3 increases 
as reaction rate increases, the majority of the reaction is completed in the beginning and is fast enough to draw enough heat to lower the temperature of the gas. Once the reaction rate starts to decrease, so does Constant 3 . The thermal conductivity of the gas mixture follows a nearly identical pattern.

The other variable that was changed to observe the results on the system was initial temperature. Most literature states an initial temperature of $500.0^{\circ} \mathrm{C}(773.15 \mathrm{~K})$, which was the initial temperature for the activation energy tests. The initial temperature was changed while now keeping activation energy constant. Temperature would now range from $650-450^{\circ} \mathrm{C}$, changing 1 degree each iteration. Towards the higher end of the range the solver encounters difficulties and going above the selected range can result in numerical issues. The conversion at these temperatures almost immediately approaches $100 \%$ conversion so going higher than the selected range

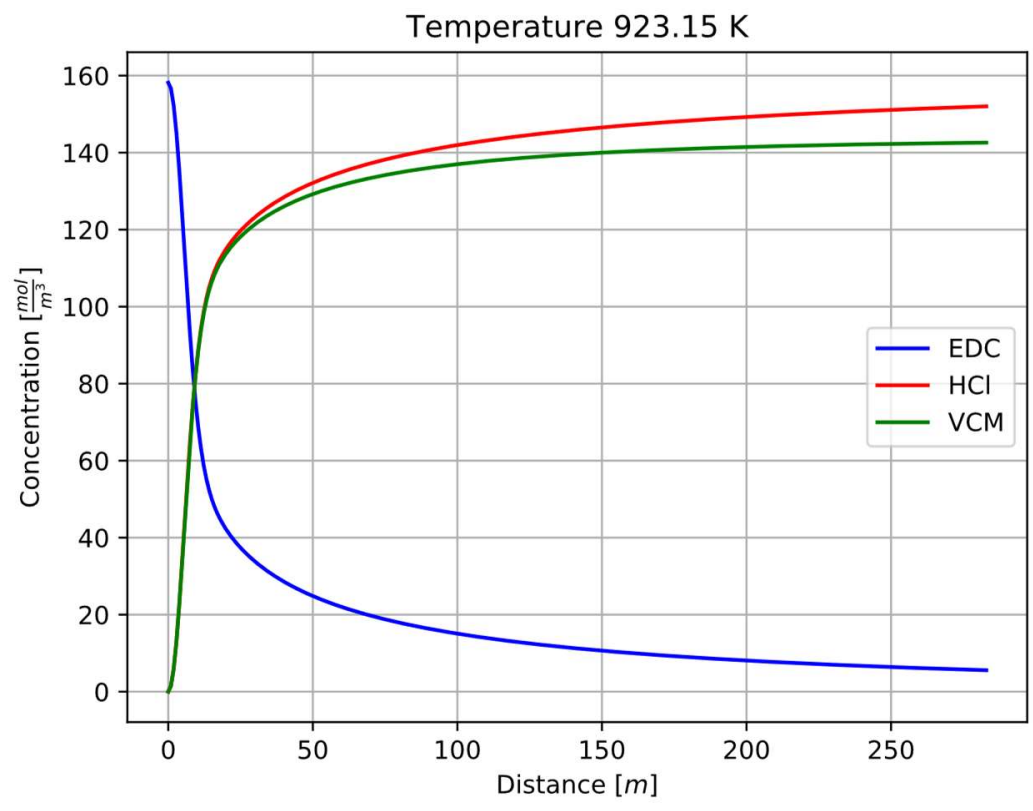

Figure 17. Product Concentration profile with an initial temperature of $923.15 \mathrm{~K}$ 
either results in failure or the results are not significantly different to warrant further modeling.

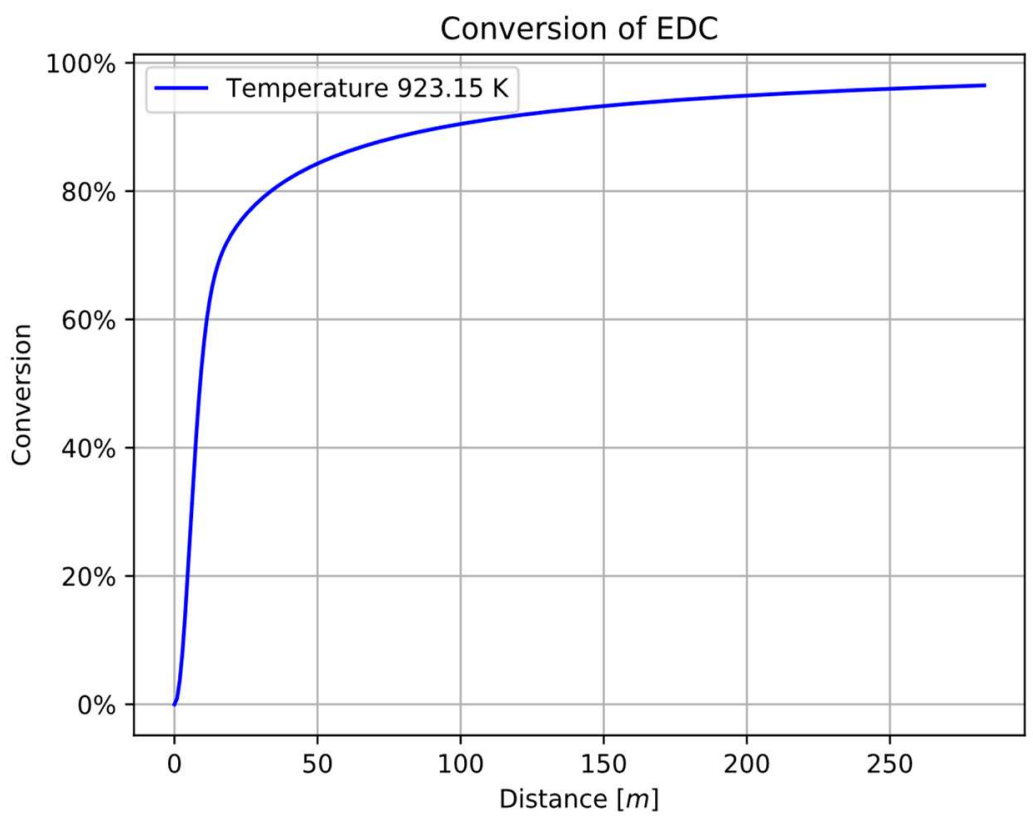

Figure 18. Conversion profile of EDC at $923.15 K$

On the lower end of the range the conversion doesn't exceed $20 \%$, as this is only 50 degrees below initial conditions, it is clear that temperature has a very important role in the reaction system. While increasing the temperature increases the conversion, also decreases the overall purity. The higher temperatures reduce the purity of the products down to approximately $93 \%$. Temperature must be carefully selected in order to achieve a desired outcome. Throughout the process the temperature of the outside wall was always set to the initial temperature of the gas feed. The relationship between temperature and conversion is shown below. Temperature appears to be linearly related to conversion, while being inversely related to purity. 


\section{Final Conversion}

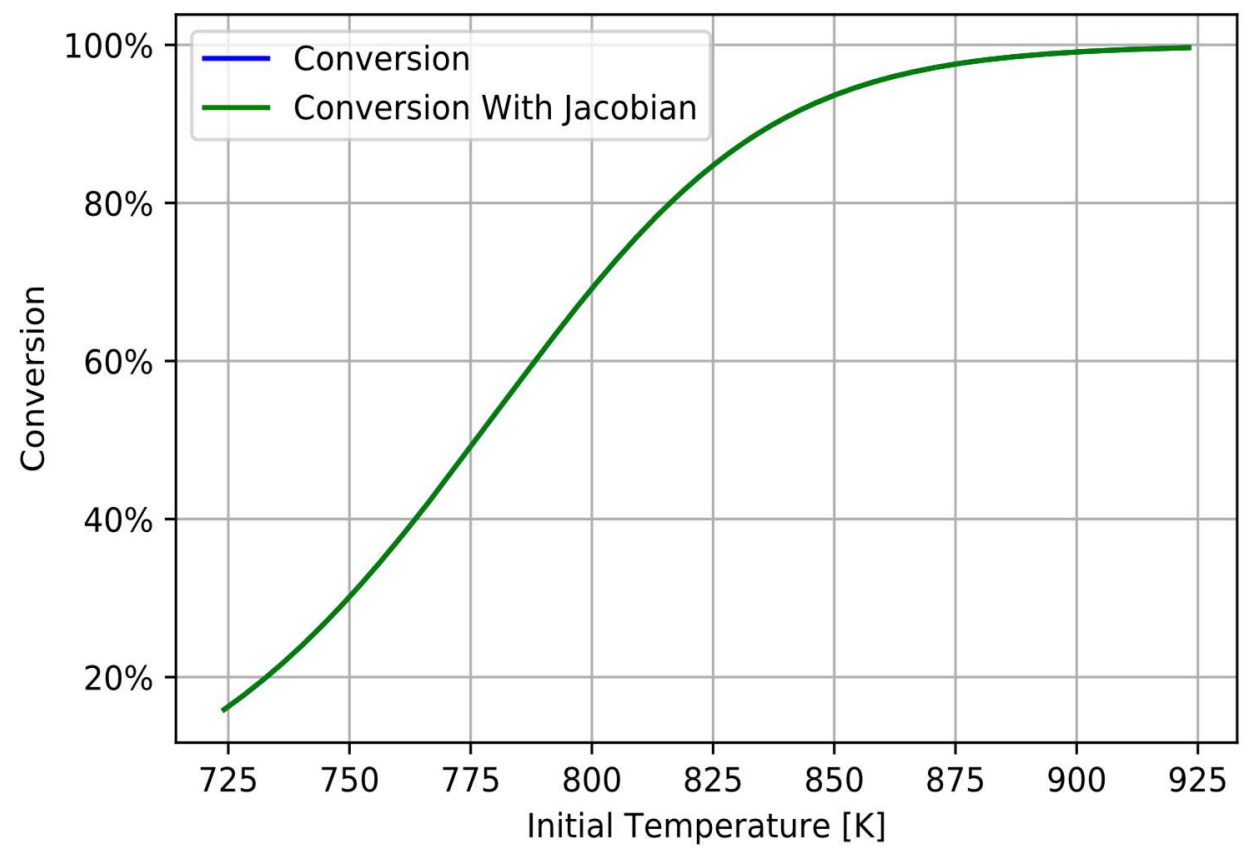

Figure 19. Final Conversion over the total range of temperature values

A large number of reactions in the system involve radicals of present species.

One of the most dominant radicals is $R_{1}$. $R_{1}$ is a negatively charged chlorine atom that is necessary to complete the formation of VCM. However, it is consumed and produced by many different reactions making it difficult to predict. Like most radicals, all radicals involved with the process are shortly consumed by other reactions. This prevents a high concentration of radicals from forming in the mixture. Of the six total radicals present at any given time, only $R_{3}$ accumulates significantly over the length of the reactor. All other radicals exist only in concentrations orders of magnitude less than $R_{3}$. There is potential to manipulate the gas mixture to produce more radicals, such as $R_{1}$, to catalyze the reaction. This could potentially increase conversion while keeping the desired level of product purity. 


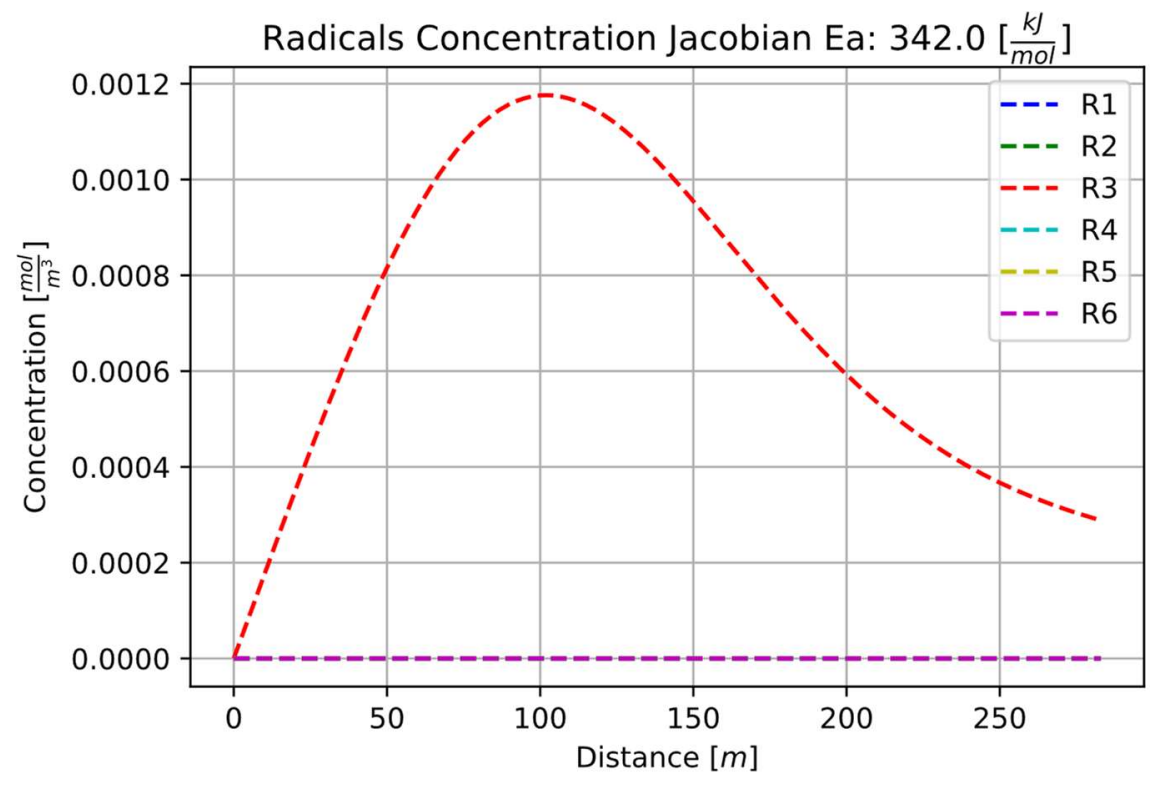

Figure 20. Concentration profile of all radical species

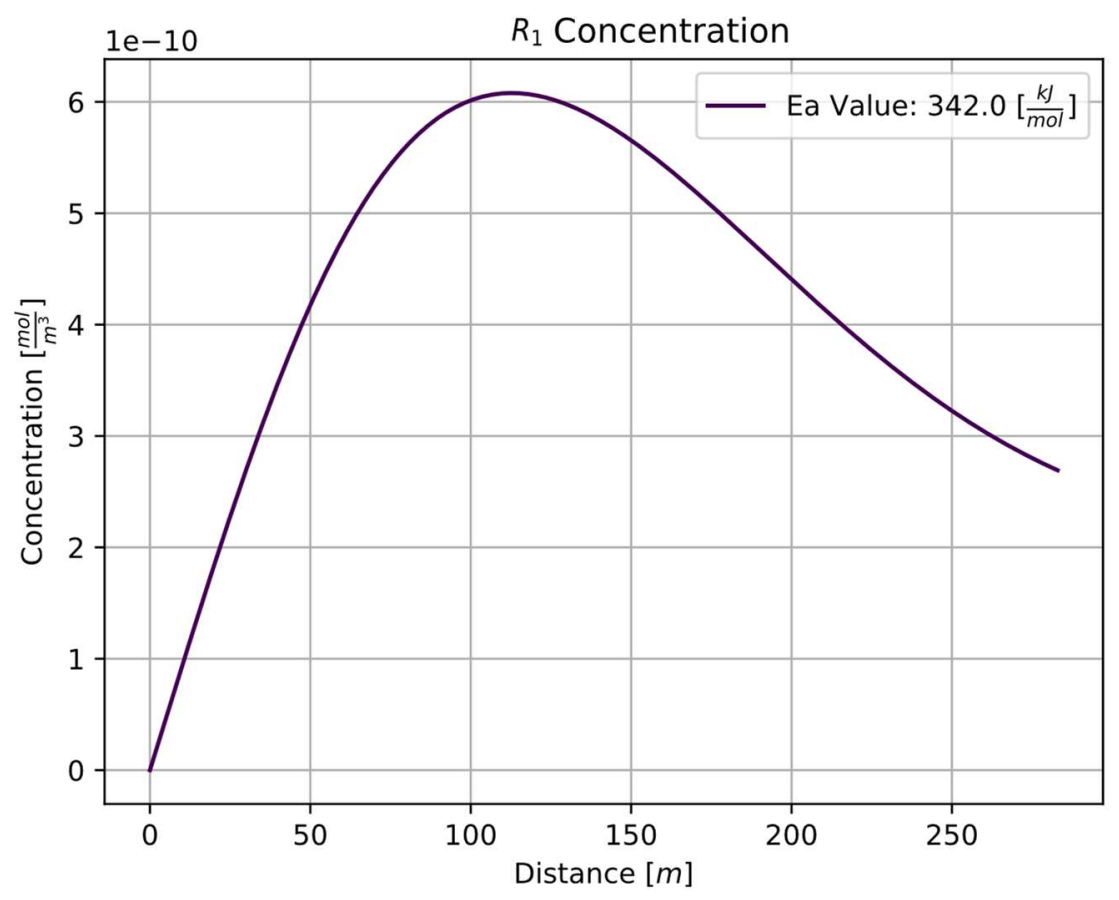

Figure 21. Concentration profile of $R 1(\mathrm{Cl}$-) 


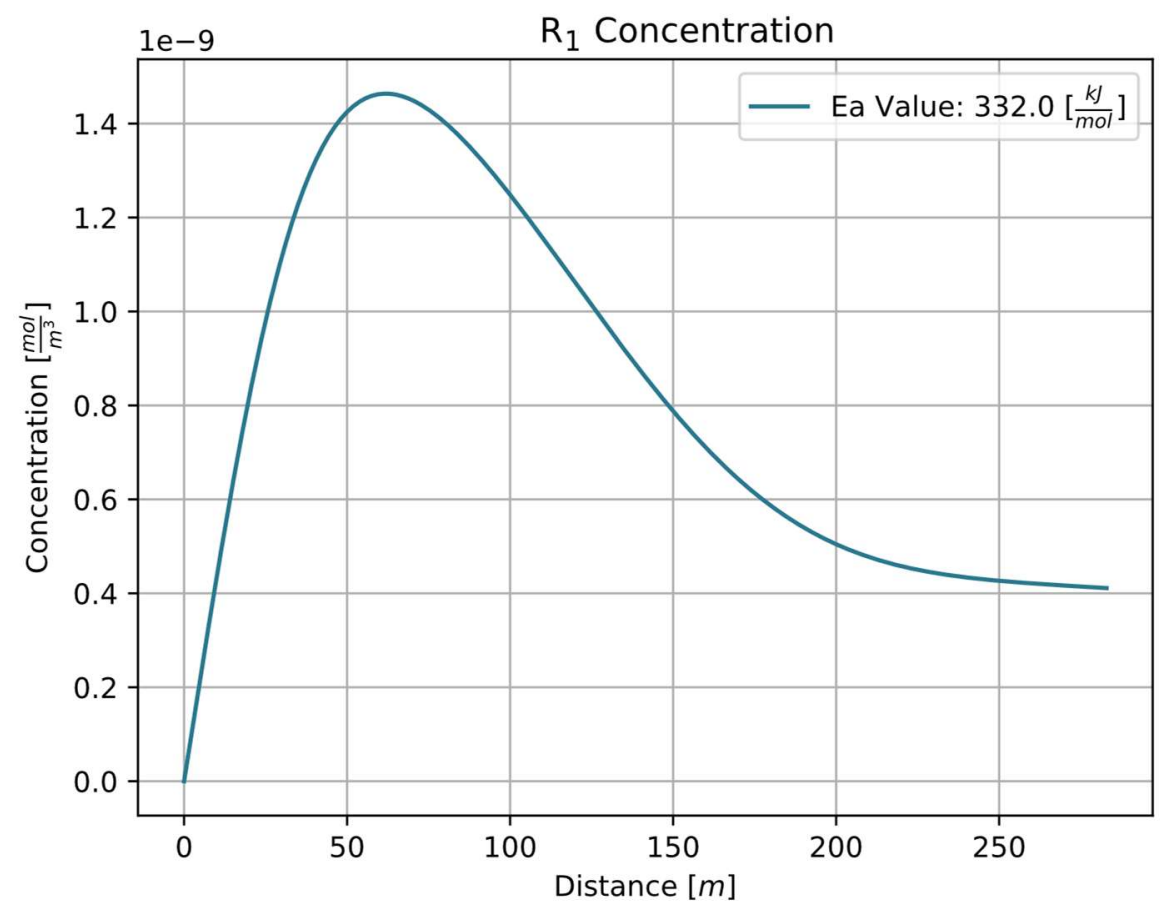

Figure 22. Concentration profile of $R 1$ at an Ea value of 332.0

The faster the reaction occurs, the higher the concentration of radicals in the mixture. This has a significant effect on the selectivity and conversion. Both temperature and activation energy affect the consumption and production of $R_{1}$. Since it is initially formed during the starting reaction, EDC's activation energy is directly related to the production of $R_{1}$. This can result in concentrations that are orders of magnitude apart, as shown below. Since lowering the activation energy doesn't have a significant affect on product purity, an increased concentration of $\mathrm{R}_{1}$ would likely assist in the conversion of EDC.

In terms of unwanted side products, there are seven main species. These species are ethyl chloride, 1,1-dichloroethane, 1,1,2-trichloroethane, 1,1-/cis-/trans- 
dichloroethylene, 1-/2-chloroprene, acetylene, benzene, and 3,4-dichlorobutene. While there is also a known "coke" material, very little information is available other than kinetic parameters. It is considered that the "Coke" material that covers the reactor as a solid cake or other places is made up of many different organic compounds making it difficult to characterize. [25] This makes calculating transport and thermodynamic properties especially difficult. [26] When this was necessary the material properties of other species in the mixture were used since no concrete value or information could be obtained. While it is ideal to avoid the production of these substances, it is generally not possible to completely prevent them. Only significant quantities of 3,4-dichlorobutene $\left(\mathrm{C}_{4} \mathrm{H}_{6} \mathrm{Cl}_{2}\right)$ was observed throughout the reaction. While none of these chemicals were ever present in significant quantities, lowering the activation energy lowers their respective concentrations. The by products build up over time and can start to foul the equipment used to sustain these reactions.

Lowering the production of the unwanted by-products can save large amounts of resources both financial and other wise. [3] A potential catalyst that is able to reduce the activation energy by $10-15 \mathrm{~kJ} / \mathrm{mol}$ would be extremely useful in industrial applications.

Once both solvers were completed and run, the activation energy solver took approximately 35 hours to complete, whereas the temperature solver took approximately 75 hours to complete. The biggest factor regarding computation time was the precision set by the "Decimal" package used. This could sometimes double or even triple the amount of time needed but was often necessary to allow the solver to 


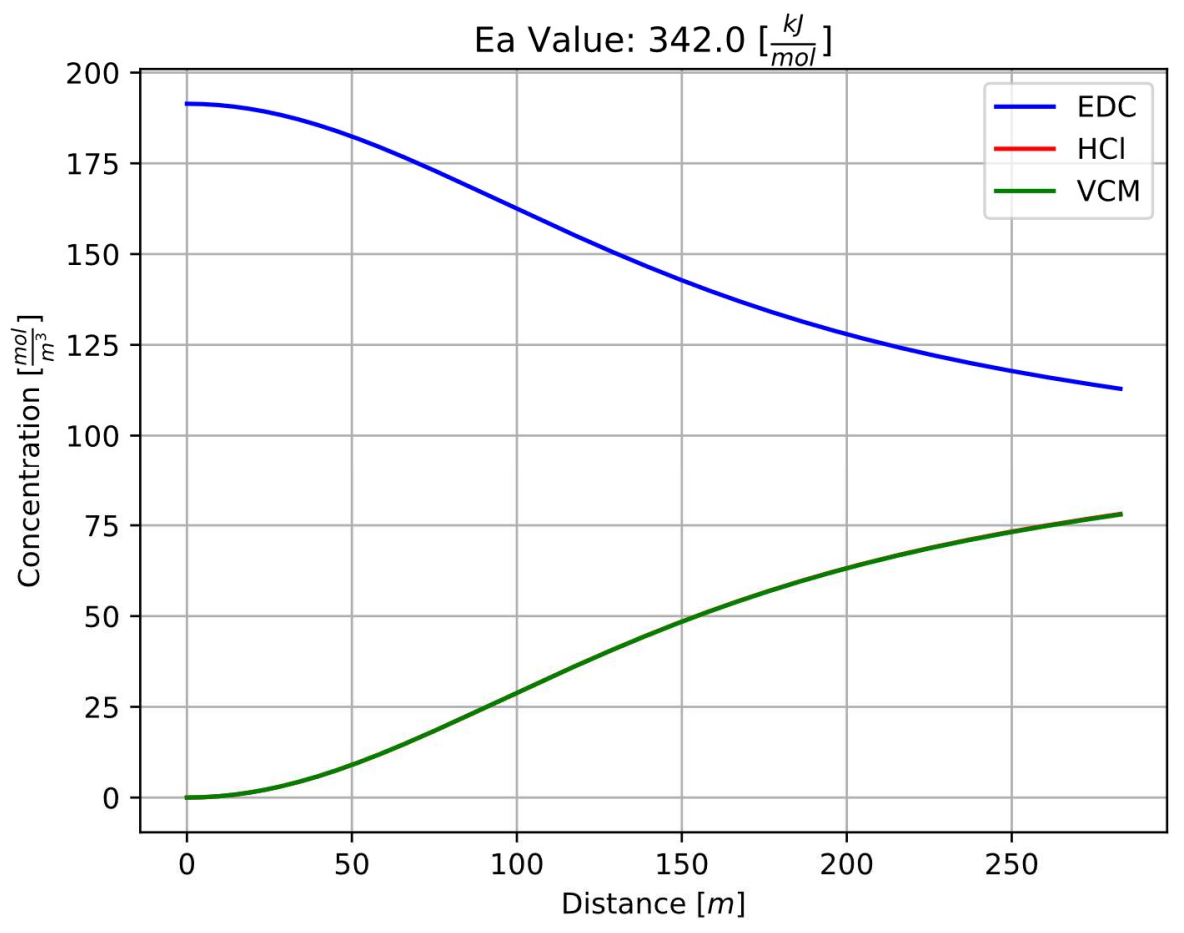

Figure 24. Product concentration profile using 300 iterations

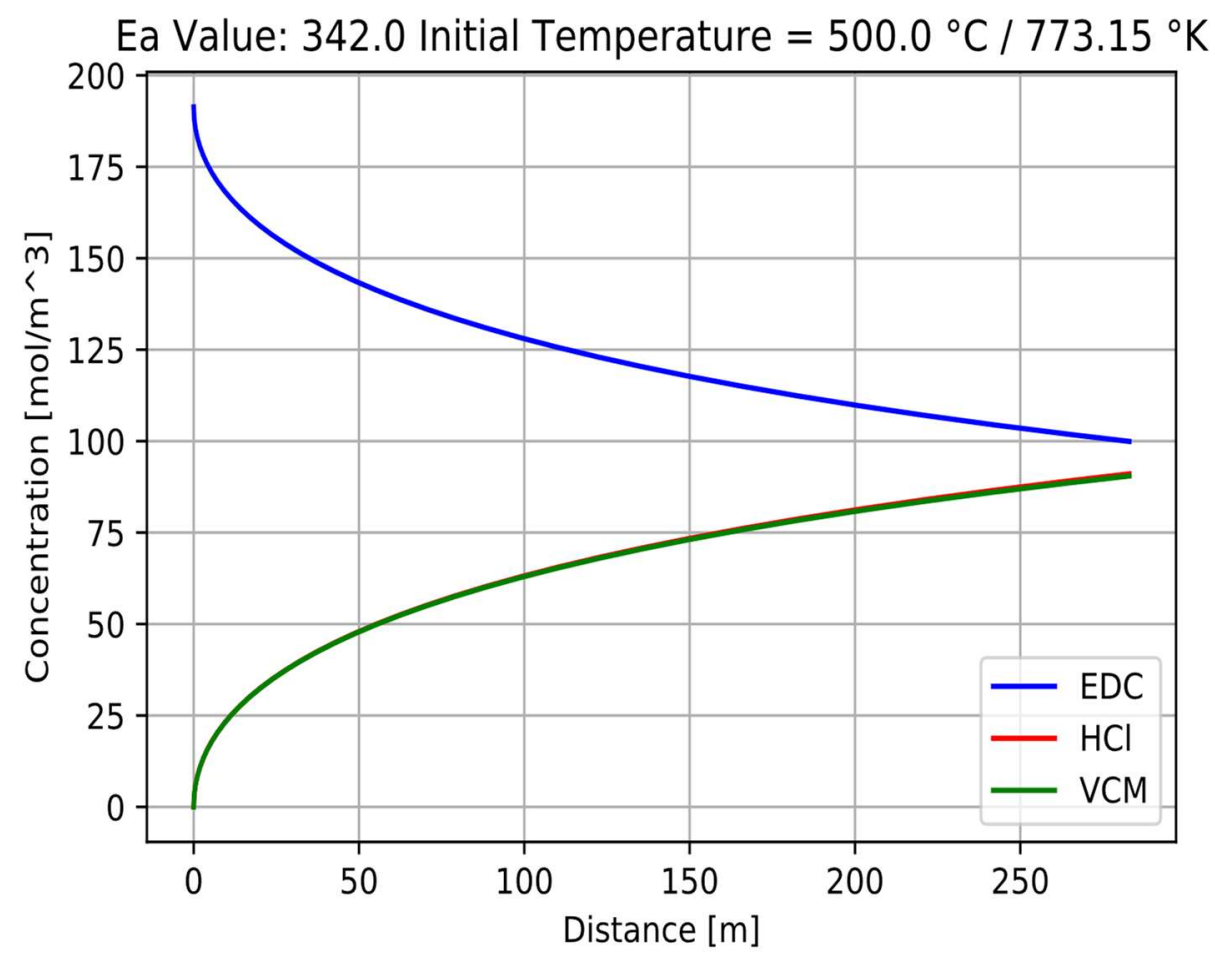

Figure 23. Product concentration profile using 3000 iterations

converge on to an answer. The number of iterations used effected the outcomes values and accuracy. For the purpose of this paper, 10 iterations per second were run to ensure 
the best possible accuracy without unreasonably long computation time. Some tests were run at 100 iterations per second. This increased the computation time up to 5 days for the activation energy solver. The results however, only differed by $1-3 \%$ from the results using 10 iterations per second.

In general, the solver with and without a Jacobian produced almost identical results most of the time. When differences appeared, they were always insignificant. At worst the difference between the two could be up to $0.5 \%$. In most case the difference is not measurable in graphs. In a few cases, specifically the temperature profile long the reactor, the was a small but noticeable difference. According to the official documentation [27], providing the Jacobian is preferred and will significantly improve accuracy and lower computational time. While the Jacobian didn't seem to have a significant effect on accuracy, it did save considerable time throughout this project.

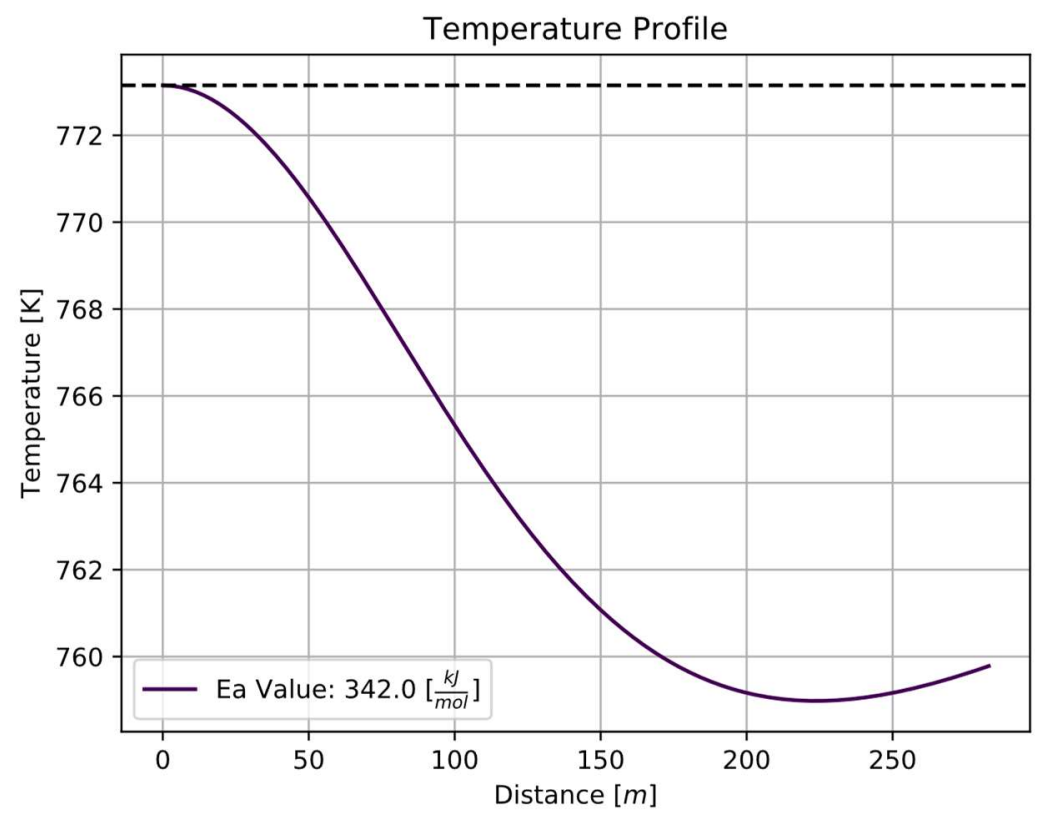

Figure 25. Temperature profile without a Jacobian 


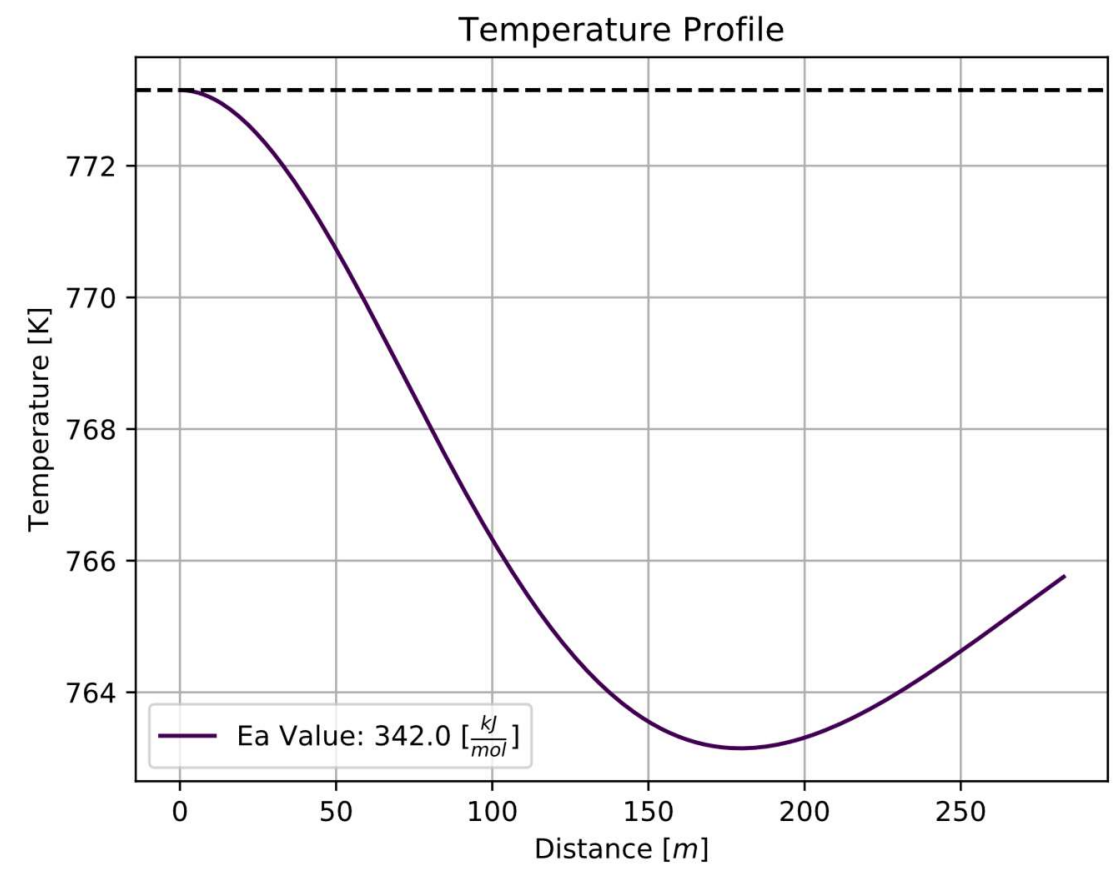

Figure 26. Temperature profile with a Jacobian

The diffusion solving method frequently ran into numerical issues and flailed, so care must be taken when considering diffusion. This is due to the fact that a large system of 38 second-order linear differential equations became too complex for the solving method to converge on reasonable answers. Since the assumption of plug flow rules out diffusion almost entirely, diffusion should not play a measurable role in the reaction process. Future work could optimize the system to function properly with the solver. As there are many components in the mixture, analytically deriving expressions for individual mass transfer equations outside of the continuity equations becomes very difficult. The second order ODE was derived correctly for the given assumptions according to [28] but the solve was unable to solve it in any way. When this system is set 
up correctly, nearly halve of the equations will initially be set to zero. This cause problems for the solver when it tries to integrate the system of ODEs. [29]

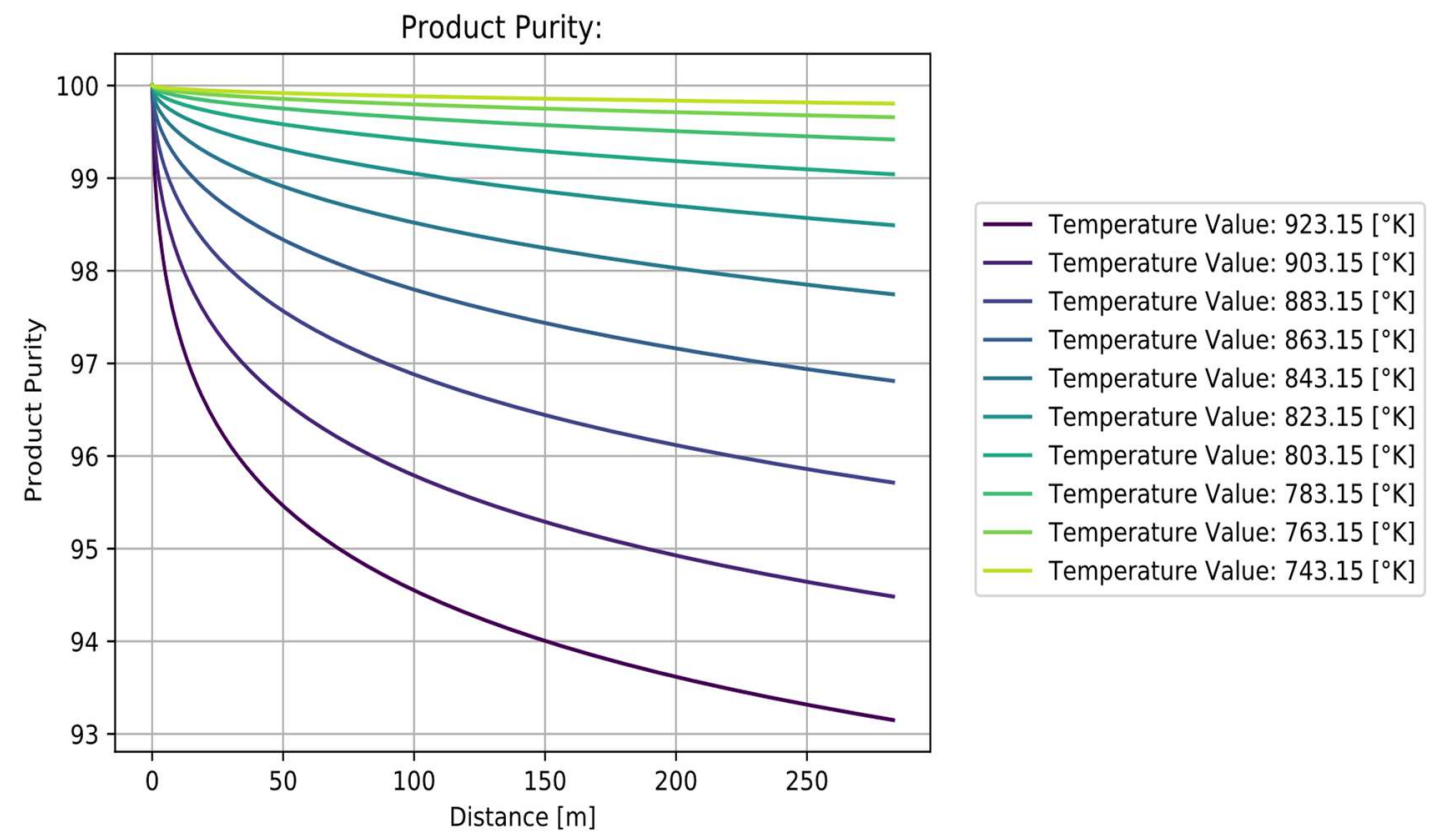

Figure 28. Product purity over the temperature range



Figure 27. Conversion of EDC over the activation energy range 
Using the specified initial condition, a overall conversion of EDC was determined to be within the range of $45-55 \%$ depending on the parameters chosen. One of the biggest changes to the system of equations given in [3] is the omission of $\mathrm{CCl}_{4} . \mathrm{CCl}_{4}$ is an extremely effective catalyst for the pyrolysis of EDC. Concentrations as low as $100 \mathrm{ppm}$ [3] can resulted in increased conversion. This omission however, was determined to cause a lower conversion by 5 - 10\%. Initial concentrations of $\mathrm{CCl}_{4}$ can make up as little as $0.1-1 \%$ [3]. When $\mathrm{CCl}_{4}$ is removed from the system, a number of dependent reactions disappear as well. This results in a system that is half the size of the one used in [3]. All side reactions created by the addition of $\mathrm{CCl}_{4}$ are almost exclusively undesired sideproducts, by removing them from the system a higher purity is achieved. Before the energy equation was coupled with all the mass balances, a solver using all of the reactions listed in [3] was built. When these builds were run to completion with an initial concentration of $0.1 \%$, the resulting values were very similar to the conversion and reaction time obtained primarily in [3]. The conversion was approximately $55-60 \%$ and the reaction time was held steady at 30 seconds. However, the effects of $\mathrm{CCl}_{4}$ was not of particular interest for this project so the simple solvers were used almost exclusively. The new solvers containing all of the aforementioned reactions are currently being built but are not yet operational.

The resulting system requires less computational time and is less likely to run into numerical issues or fail. Originally both systems were built, but after the simpler list was coupled with the energy balance, all experiments were run using the simpler 
system. Even the system is shorter and gives a lesser conversion, the overall theories and concepts its meant to understand and experiment with stay the same. The same proportion of conversion increases and decreases with a respective change in either temperature or activation energy. This allows it to be used for the same purposes as the larger system. 


\section{Conclusion}

The roles, concepts, and evaluation of temperature and activation energy have been thoroughly investigated using the created solvers. Their effect on the outcome and performance of the overall system within the specific range has been established. How variables such as conversion, product purity, and operation parameters change in respect to a change in either of the two independent variables. Solver conditions have been optimized to run as efficiently and accurately as possible within the given bounds. It should be noted however that going out of the given range for either of the two variables may cause the solver to fail.

In terms of activation energy, the given range was $342.0-317.0 \frac{\mathrm{kJ}}{\mathrm{mol}}$. A total of 100 iterations were performed over the entire range, lowering the activation energy by a value of $0.25 \frac{\mathrm{kJ}}{\mathrm{mol}}$ per iteration. For simple reactions it can generally be theorized that lowering the activation energy for an endothermic reaction will increase the conversion all else held constant. For much more complex systems, especially ones with indirect pathways to the desired product, it cannot always be assumed to be true. Through numerical integration, it was determined that the overall conversion does in fact increase by lowering the activation energy. 
By lowering the initial activation energy by $10-15 \frac{\mathrm{kJ}}{\mathrm{mol}}$ the conversion is increased by approximately $15-20 \%$, sometimes higher. While the conversion increases, the product purity is not significantly changed with respect to its initial values, generally maintaining a value of $97 \%$ or higher. This demonstrates great potential for a catalyst as it could potentially greatly improve the existing process without much of a drawback.

Decreasing the activation energy by more than $15 \frac{\mathrm{kJ}}{\mathrm{mol}}$ will put the conversion higher, with the lowest value of $317.0 \frac{\mathrm{kJ}}{\mathrm{mol}}$ producing a conversion of around $92 \%$. If the conversion gets too close to $100 \%$ conversion, the solver will run into numerical issues and fail.

In terms on initial temperature of the gas feed.an increase in temperature cause an increase in conversion, while decreasing the temperature decreases the conversion. It was evaluated over 200 iterations over the range of $650-450{ }^{\circ} \mathrm{C}$. Each iteration would lower the temperature by exactly 1 degree. The temperature of the outside wall was held constant at the initial temperature of the gas feed. Unlike activation energy, when the conversion increase is due to temperature, the purity of the products does decrease. The highest temperature value of $650^{\circ} \mathrm{C}$ results in a product purity of less than $93 \%$ whereas the lowest $450{ }^{\circ} \mathrm{C}$ results in a product purity of above $99 \%$. As temperature affects every species in the entire system unlike activation energy, the solver for temperature is much more susceptible to numerical issues than the activation energy solver. All of the presently used solvers assumed the omission of $\mathrm{CCl}_{4}$ and its respective reactions. 
This project has demonstrated that the reduction in activation energy produces results desirable for improving the overall process to manufacture VCM. The results strongly indicate a catalyst capable of reducing the activation energy by $10-15 \frac{\mathrm{kJ}}{\mathrm{mol}}$ would significantly improve conversion while maintaining the desired level of product purity. This catalyst could also decrease the temperature necessary to achieve $60 \%$ conversion, increasing the product purity even higher. Industrial size production of VCM could reduce cost, improve safety, and become more environmentally friendly.

There are many things that could be considered for future work. The first thing is to build upon the simple model solver to incorporate all reactions listed in [3]. From experience with earlier attempts it seems best to make them different solvers and not incorporate them into the same program. Even if the amount of $\mathrm{CCl}_{4}$ is entered as zero, the solving methods use can still sometimes return values that should be exactly zero as numbers very close to zero, both positive and negative. For this reason, the" RHS" function for both solvers should be completely separate. Once it is completed the system parameters will have to be optimized for the larger system. Based on experience this shouldn't be too difficult but will be somewhat time consuming. It will also be important to select a reasonable value for the initial $\mathrm{CCl}_{4}$. The new solvers could be used in conjunction with the simple solvers to measure the effect of $\mathrm{CCl}_{4}$ on the system directly.

Once both systems are fully operational there may be interest in improving the model in one or both of the following way. The first is adding the second order ODEs for 
each respective mass component. Once properly executed the available solvers can not properly integrate the resulting system due to a numerical issue with the resulting system. This is further detailed in [29]. This would allow the solvers to function without the assumption of plug flow, expanding the scope and application of the solvers.

Next is to transform the system of ODEs currently used to a system of PDEs to incorporate the radial dimension in the equation. This may prove difficult as the reaction term for the majority of the chemical species is to large to properly fit into most available PDE solvers. Due to the assumptions of turbulent flow and plug flow, both of these improvements may not have any significant effect on the results obtained from the solver. These improvements however, would allow these solvers to be used in situations outside of a PFR with plug flow. 


\section{References}

1. Schirmeister, R., J. Kahsnitz, and M. Träger, Influence of EDC Cracking Severity on the Marginal Costs of Vinyl Chloride Production. Industrial \& Engineering Chemistry Research, 2009. 48(6): p. 2801-2809.

2. Borsa, A.G., Industrial Plant/Laboratory Investigation and Computer Modeling of 1,2-Dihloroethane Pyrolysis. 1999, Colorado School of Mines: Ann Arbor. p. 236.

3. Hirschfelder, J. O. (1963), "Applied Mathematics as used in Theoretical Chemistry", American Mathematical Society Symposium: 367-376.

4. Lakshmanan, A., W.C. Rooney, and L.T. Biegler, A case study for reactor network synthesis: the vinyl chloride process. Computers \& Chemical Engineering, 1999. 23(4): p. 479-495.

5. Nyeng I (2015) Modeling of a 1,2-Dichloroethane Cracker. Norwegian University of Science and Technology. Department of Chemical Engineering

6. Fogler, H. S. (2006). Elements of chemical reaction engineering (4th ed.).

7. Xun, Shen \& Shaojun, Xia \& Fengrui, Sun. (2017). Thermodynamic analyses for recovering waste heat of high-temperature basic oxygen gas (BOG) by the methane reforming with carbon dioxide reaction. Energy. 118. 906-91.

8. Weisstein, Eric W. "Ordinary Differential Equation Order." From MathWorld--A Wolfram Web Resource.

9. Bird, R B, Warren E. Stewart, and Edwin N. Lightfoot. Transport Phenomena. New York: J. Wiley, 2007. Print.

10. Zhuang, P., et al. "Numerical Methods for the Variable-Order Fractional Advection-Diffusion Equation with a Nonlinear Source Term." SIAM Journal on Numerical Analysis 47.3 (2009): 1760-22. ProQuest. Web. 9 Oct. 2019.

11. Davis, Mark E. and Davis, Robert J. (2003) Fundamentals of chemical reaction engineering. McGraw-Hill chemical engineering series. McGraw-Hill Higher Education, New York, NY. ISBN 007245007X.

12. Incropera, Frank P, and David P. DeWitt. Fundamentals of Heat and Mass Transfer. New York: J. Wiley, 2002. Print.

13. Bell, C., Chemical properties component of Chemical Engineering Design Library (ChEDL). 2016: https://github.com/CalebBell/thermo.

14. Kim, Sun Ung, and Charles W. Monroe. "High-Accuracy Calculations of Sixteen Collision Integrals for Lennard-Jones (12-6) Gases and Their Interpolation to Parameterize Neon, Argon, and Krypton." Journal of Computational Physics 273 (September 15, 2014): 358-73. doi:10.1016/j.jcp.2014.05.018.

15. Fairbanks, D. F. and C. R. Wilke (1950). "Diffusion Coefficients in Multicomponent Gas Mixtures." Industrial \& Engineering Chemistry 42(3): 471-475.

16. Sutton K, Gnoffo PA. 1998. Multi-component diffusion with application to computational aerothermodynamics. AIAA Pap. 98-2575

17. Poling, Bruce E. The Properties of Gases and Liquids. 5th edition. New York: McGraw-Hill Professional, 2000Lastovka, Vaclav, and John M. Shaw. "Predictive Correlations for Ideal Gas Heat Capacities of Pure Hydrocarbons and Petroleum Fractions." Fluid Phase Equilibria 356 (October 25, 2013): 338-370. doi:10.1016/j.fluid.2013.07.023

18. Lindsay, Alexander L., and LeRoy A. Bromley. "Thermal Conductivity of Gas Mixtures." Industrial \& Engineering Chemistry 42, no. 8 (August 1, 1950): 1508-11. doi:10.1021/ie50488a017

19. Danner, Ronald P, and Design Institute for Physical Property Data. Manual for Predicting Chemical Process Design Data. New York, N.Y, 1982.

20. Robert W. Serth, Thomas G. Lestina, Process heat transfer principles, applications and rules of thumb, Second edition 2014, Elsevier Inc. 
21. Brokaw, R. S. "Predicting Transport Properties of Dilute Gases." Industrial \& Engineering Chemistry Process Design and Development 8, no. 2 (April 1, 1969): 240-53. doi:10.1021/i260030a015.

22. Buddenberg, J. W. ; and Wilke, C. R. : Calculation of Gas Mixture Viscosities. Ind. Eng. Chem., vol. 41, no. 7, July 1949, pp. 1345-1347.

23. E. Hairer, G. Wanner, "Solving Ordinary Differential Equations II: Stiff and Differential-Algebraic Problems", Sec. IV.8.

24. L. Petzold, "Automatic selection of methods for solving stiff and nonstiff systems of ordinary differential equations", SIAM Journal on Scientific and Statistical Computing, Vol. 4, No. 1, pp. 136-148, 1983.

25. Borsa, Alessandro G., et al. "Characterization of coke formed in vinyl chloride manufacture." Industrial \& engineering chemistry research 38.11 (1999): 4259-4267.

26. Coke Produced in the Commercial Pyrolysis of Ethylene Dichloride into Vinyl Chloride Isao Mochida, ${ }^{*},+$, Tadanori Tsunawaki, $\dagger$, Chiaki Sotowa, $\dagger$, Yozo Korai, $\dagger$ and, and Kazuo Higuchi Industrial \& Engineering Chemistry Research 199635 (10), 3803-3807 DOI: 10.1021/ie9600248

27. Pauli Virtanen, Ralf Gommers, Travis E. Oliphant, Matt Haberland, Tyler Reddy, David Cournapeau, Evgeni Burovski, Pearu Peterson, Warren Weckesser, Jonathan Bright, Stéfan J. van der Walt, Matthew Brett, Joshua Wilson, K. Jarrod Millman, Nikolay Mayorov, Andrew R. J. Nelson, Eric Jones, Robert Kern, Eric Larson, CJ Carey, İlhan Polat, Yu Feng, Eric W. Moore, Jake VanderPlas, Denis Laxalde, Josef Perktold, Robert Cimrman, lan Henriksen, E.A. Quintero, Charles R Harris, Anne M. Archibald, Antônio H. Ribeiro, Fabian Pedregosa, Paul van Mulbregt, and SciPy 1.0 Contributors. (2019) SciPy 1.0Fundamental Algorithms for Scientific Computing in Python. preprint arXiv:1907.10121

28. Giuliano, A. \& Pellegrino, E.. (2019). Numerical integration strategies of PFR dynamic models with axial dispersion and variable superficial velocity: the case of $\mathrm{CO} 2$ capture by a solid sorbent. Heliyon. 5 . e02040. 10.1016/j.heliyon.2019.e02040.

29. Shiraishi, Fumihide. (2001). Highly accurate solution of the axial dispersion model expressed in Ssystem canonical form by Taylor series method. Chemical Engineering Journal - CHEM ENG J. 83. 175183. 10.1016/S1385-8947(00)00252-7. 


\section{Appendices}

Simple System mass and energy balance equations:

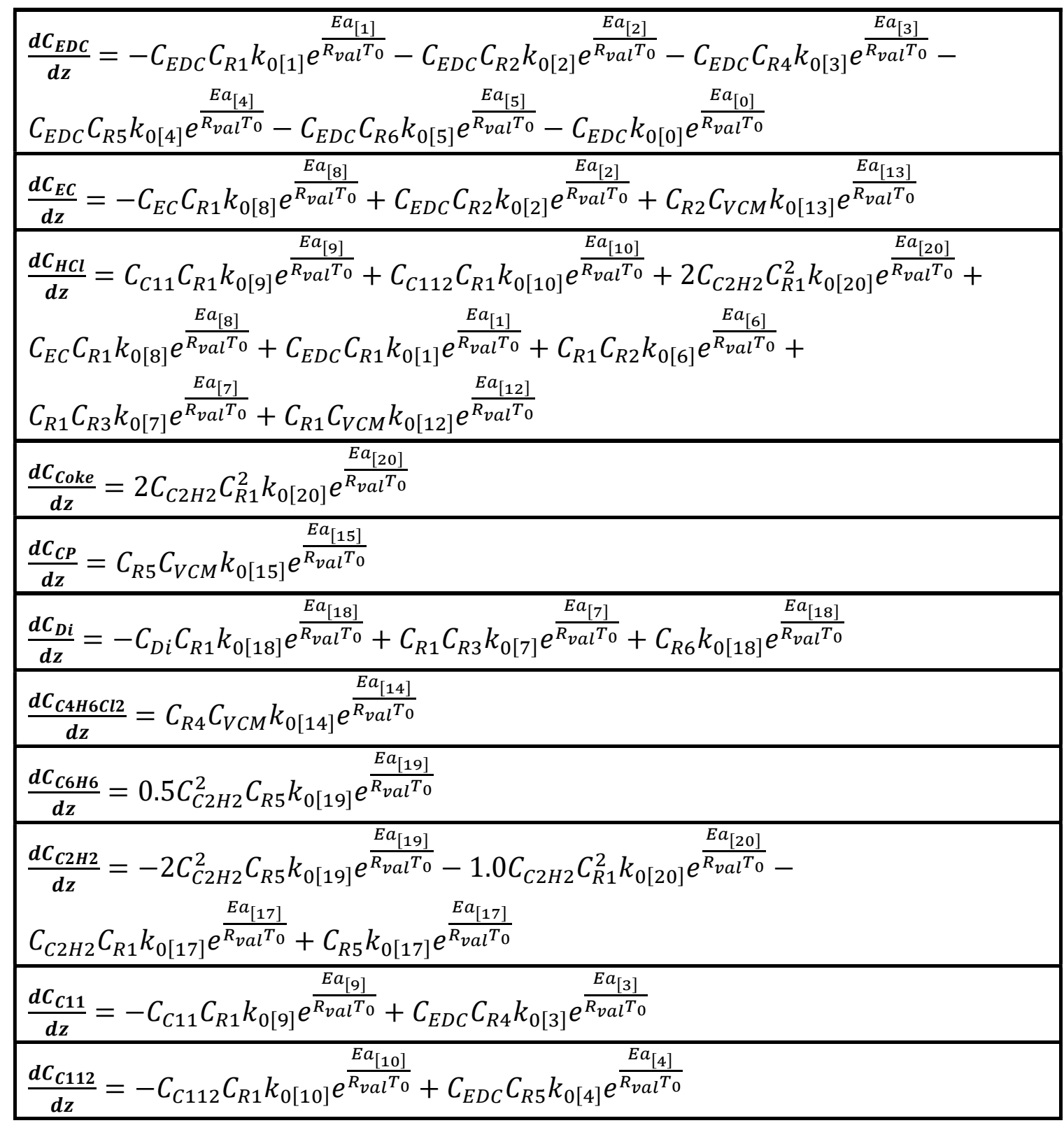




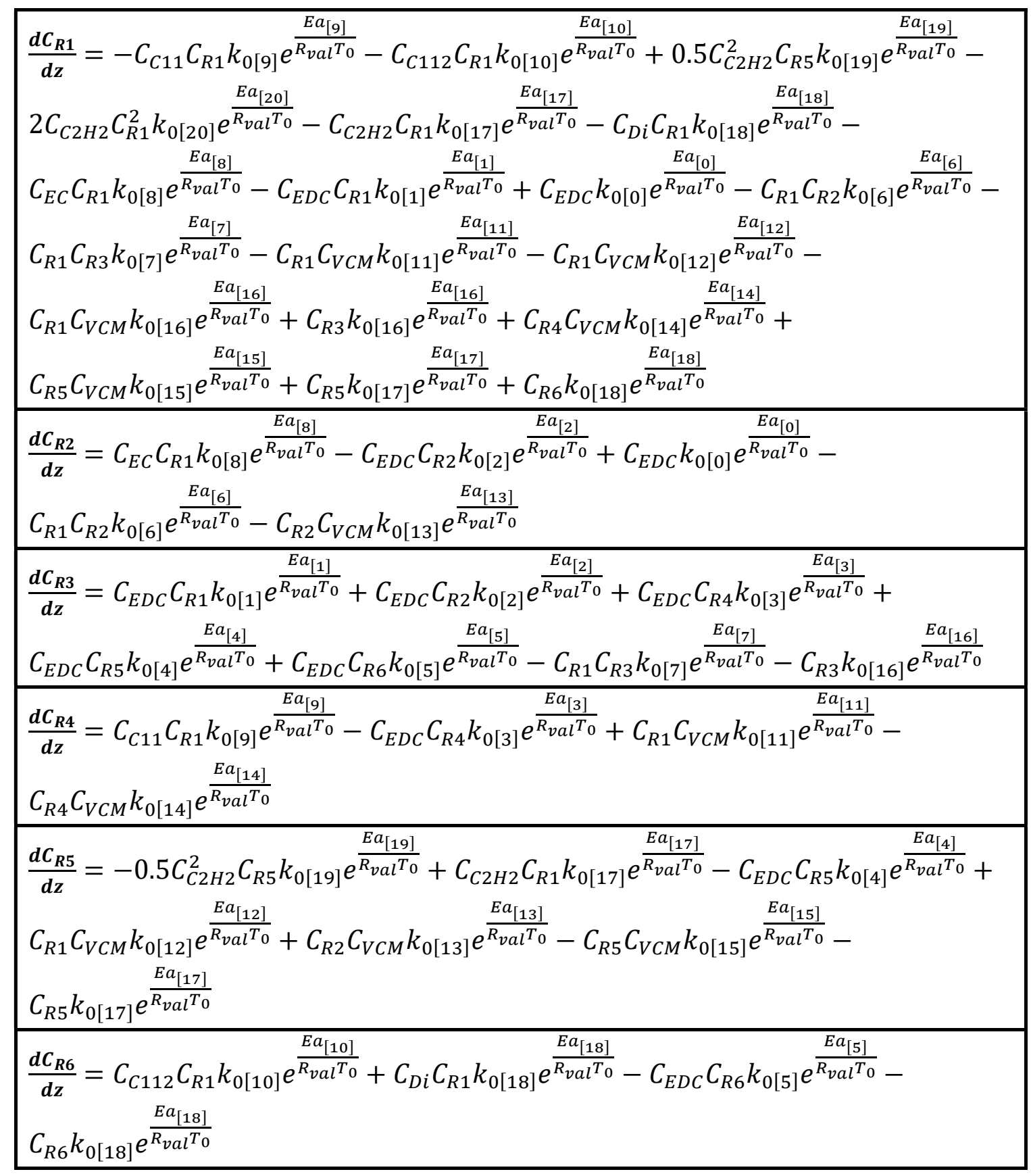




\begin{tabular}{|l|}
$\frac{d C_{V C M}}{\boldsymbol{d z}}=C_{E D C} C_{R 5} k_{0[4]} e^{\frac{E a_{[4]}}{R_{v a l} T_{0}}}+C_{R 1} C_{R 2} k_{0[6]} e^{\frac{E a_{[6]}}{R_{v a l} T_{0}}}-C_{R 1} C_{V C M} k_{0[11]} e^{\frac{E a_{[11]}}{R_{v a l} T_{0}}}-$ \\
$C_{R 1} C_{V C M} k_{0[12]} e^{\frac{E a_{[12]}}{R_{v a l} T_{0}}}-C_{R 1} C_{V C M} k_{0[16]} e^{\frac{E a_{[16]}}{R_{v a l} T_{0}}}-C_{R 2} C_{V C M} k_{0[13]} e^{\frac{E a_{[13]}}{R_{v a l} T_{0}}}+$ \\
$C_{R 3} k_{0[16]} e^{\frac{E a_{[16]}}{R_{v a l} T_{0}}}-C_{R 4} C_{V C M} k_{0[14]} e^{\frac{E a_{[14]}}{R_{v a l} T_{0}}}-C_{R 5} C_{V C M} k_{0[15]} e^{\frac{E a_{[15]}}{R_{v a l} T_{0}}}$ \\
\hline$\frac{d T}{d z}=T_{1}$ \\
$\frac{\boldsymbol{d}^{2} T}{d \mathbf{z}^{2}}=T_{1} \operatorname{con}_{1}-\left[\operatorname{con}_{2}\left(T_{w a l l}-T_{0}\right)+\operatorname{con}_{3} r_{E D C}\right]$ \\
\hline
\end{tabular}

Full system mass and energy balance equations:

\begin{tabular}{|c|}
\hline $\begin{array}{l}\frac{\boldsymbol{d} C_{E D C}}{\boldsymbol{d z}}=\left[-C_{E D C} C_{R 1} k_{0[2]} e^{\frac{E a_{[2]}}{R_{\text {val }} T_{0}}}-C_{E D C} C_{R 2} k_{0[3]} e^{\frac{E a_{[3]}}{R_{v a l} T_{0}}}-C_{E D C} C_{R 4} k_{0[4]} e^{\frac{E a_{[4]}}{R_{\text {val }} T_{0}}}-\right. \\
C_{E D C} C_{R 5} k_{0[5]} e^{\frac{E a_{[5]}}{R_{v a l} T_{0}}}-C_{E D C} C_{R 6} k_{0[6]} e^{\frac{E a_{[6]}}{R_{v a l} T_{0}}}-C_{E D C} C_{R 7} k_{0[7]} e^{\frac{E a_{[7]}}{R_{v a l} T_{0}}}- \\
\left.C_{E D C} C_{R 8} k_{0[8]} e^{\frac{E a_{[8]}}{R_{\text {val }} T_{0}}}-C_{E D C} k_{0[0]} e^{\frac{E a_{[0]}}{R_{v a l} T_{0}}}\right]\end{array}$ \\
\hline$\frac{\boldsymbol{d} C_{E C}}{\boldsymbol{d z}}=\left[-C_{E C} C_{R 1} k_{0[11]} e^{\frac{E a_{[11]}}{R_{v a l} T_{0}}}+C_{E D C} C_{R 2} k_{0[3]} e^{\frac{E a_{[3]}}{R_{v a l} T_{0}}}+C_{R 2} C_{V C M} k_{0[18]} e^{\frac{E a_{[18]}}{R_{v a l} T_{0}}}\right]$ \\
\hline $\begin{array}{l}\frac{d C_{H C l}}{d z}=\left[C_{C 11} C_{R 1} k_{0[12]} e^{\frac{E a_{[12]}}{R_{v a l} T_{0}}}+C_{C 1112} C_{R 1} k_{0[14]} e^{\frac{E a_{[14]}}{R_{v a l} T_{0}}}+C_{C 112} C_{R 1} k_{0[13]} e^{\frac{E a_{[13]}}{R_{v a l} T_{0}}}+\right. \\
2 C_{C 2 H 2} C_{R 1}^{2} k_{0[30]} e^{\frac{E a_{[30]}}{R_{v a l} T_{0}}}+C_{C H C l 3} C_{R 1} k_{0[15]} e^{\frac{E a_{[15]}}{R_{v a l} T_{0}}}+C_{E C} C_{R 1} k_{0[11]} e^{\frac{E a_{[11]}}{R_{v a l} T_{0}}}+ \\
C_{E D C} C_{R 1} k_{0[2]} e^{\frac{E a_{[2]}}{R_{v a l} T_{0}}}+C_{R 1} C_{R 2} k_{0[9]} e^{\frac{E a_{[9]} R_{v a l}}{R_{0}}}+C_{R 1} C_{R 3} k_{0[10]} e^{\frac{E a_{[10]}}{R_{v a l} T_{0}}}+ \\
\left.C_{R 1} C_{V C M} k_{0[17]} e^{\frac{E a_{[11]}}{R_{v a l} T_{0}}}\right]\end{array}$ \\
\hline$\frac{d C_{\text {Coke }}}{\boldsymbol{d z}}=\left[2 C_{C 2 \mathrm{H} 2} C_{R 1}^{2} k_{0[30]} e^{\frac{E a_{[30]}}{R_{\text {val }} T_{0}}}\right]$ \\
\hline$\frac{d C_{C P}}{d z}=\left[C_{R 5} C_{V C M} k_{0[20]} e^{\frac{E a_{[20]}}{R_{v a l} T_{0}}}\right]$ \\
\hline
\end{tabular}









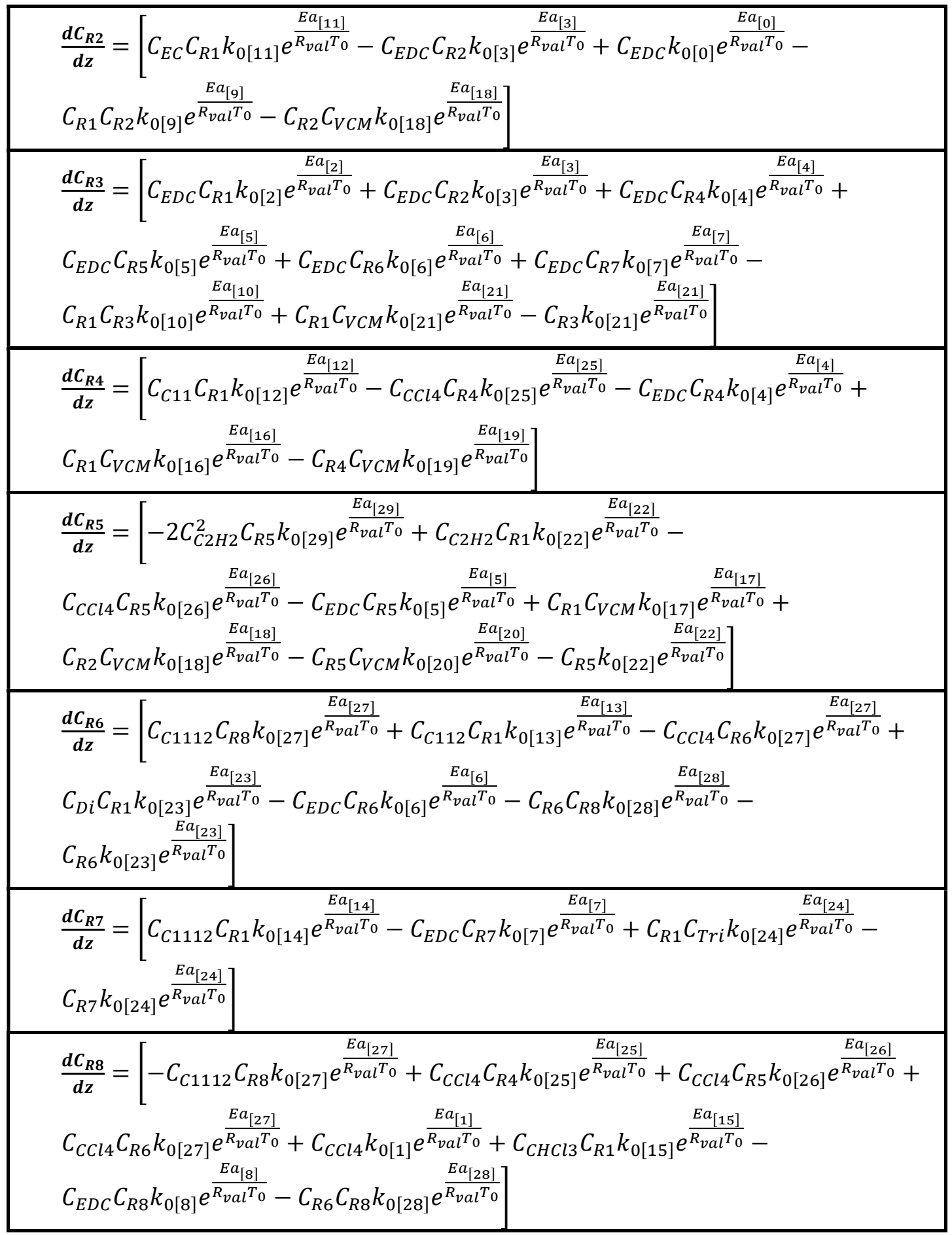









\title{
CURRICULUM VITA
}

\author{
NAME: $\quad$ Travis James Czechorski \\ ADDRESS: $\quad 1783$ Stephenson mill road \\ Verona, KY, 41092. \\ DOB: $\quad$ Bedford, Texas - January 26, 1995 \\ EDUCATION \\ \& TRAINING: B.S., Chemical Engineering \\ University of Louisville \\ 2013-2018
}

